\title{
استخدام أساليب السلاسل الزمنية لمعالجة الاختلافات الموسمية في الرقم القياسي لسعر المتهلك
}

\author{
الباحث/ هيثم حسون ماجد
}

أ. م. د. عبد اللطيف حسن شومان

المستخلص مو متر

كما هو معروف أن الرقم القياسي لسعر المستهلك (CPI) هو احد الهم الأرقام القياسية المستخدمة

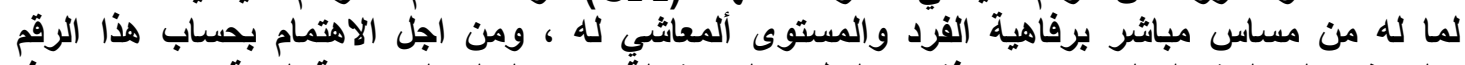

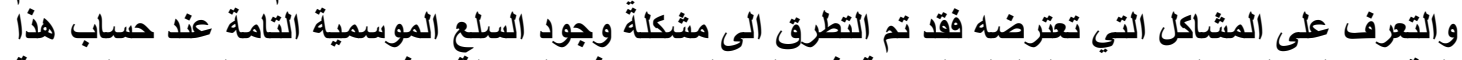

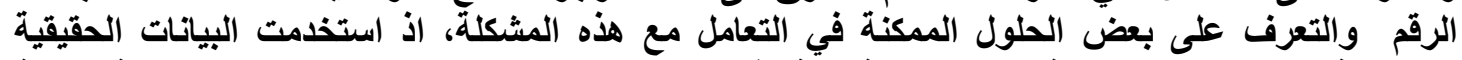

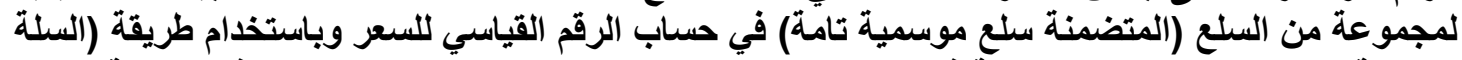

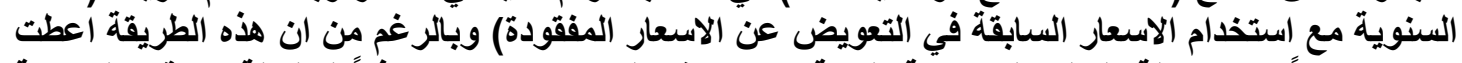

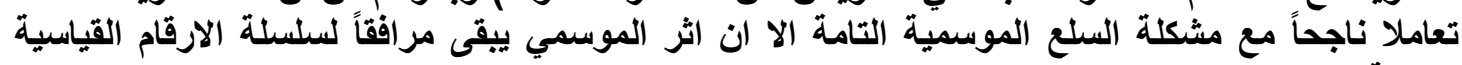

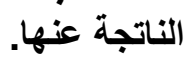

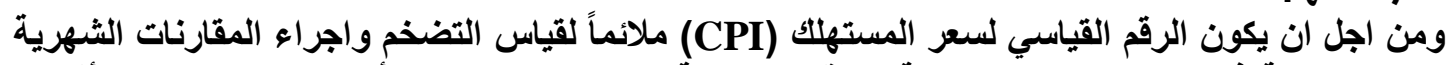

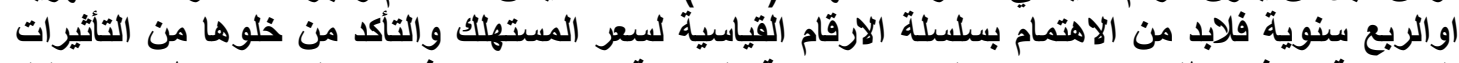

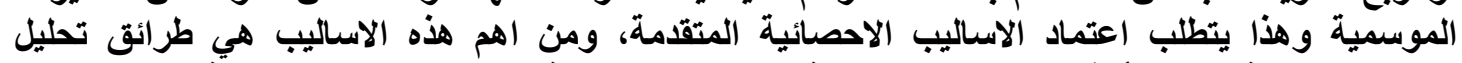

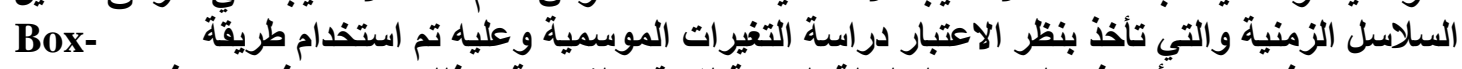

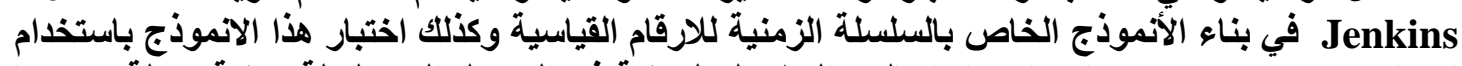

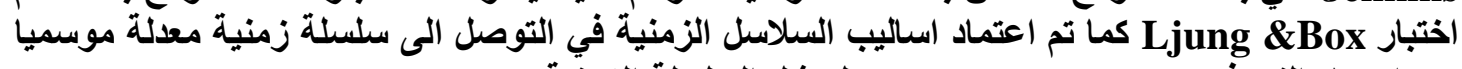

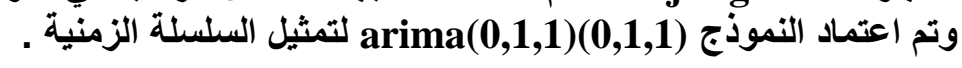

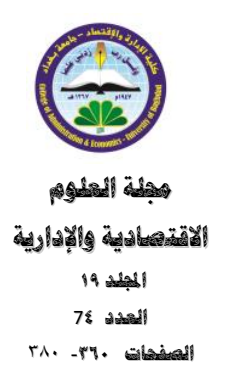

البحث مستل من رسالة ماجستير

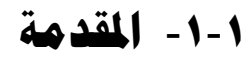


يعد الرقم القياسي لسعر المستهلك (Consumer Price index) من الأرقام القياسية المهمة لما له

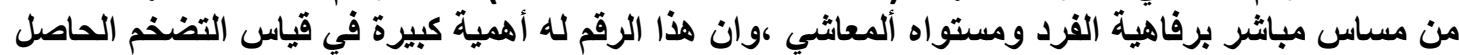

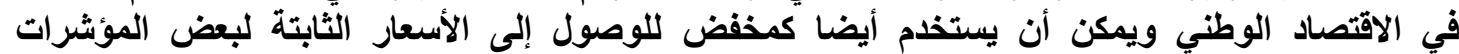

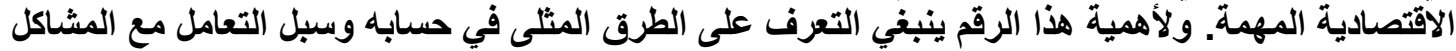

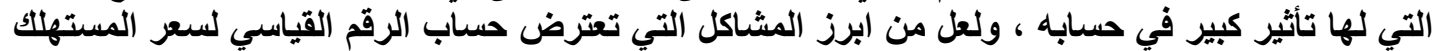

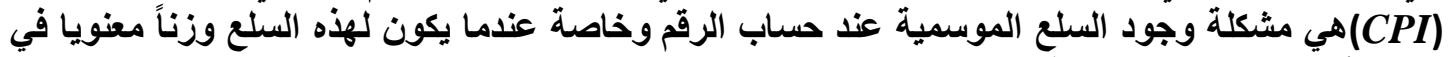

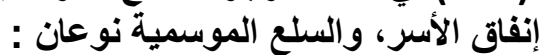

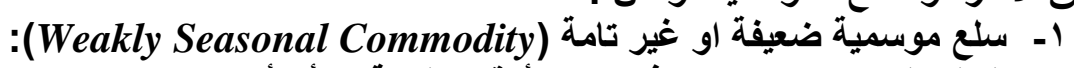

وهي السلع التي تكون موجودة في جميع أوقات السنة إلا أن أسعار ها وكمياتها تخضع لتقلبات منتظمة مر افقة لمواسم السنية.

r- سلع موسمية تامة (قوية) (Strongly Seasonal Commodity):

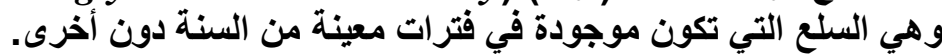

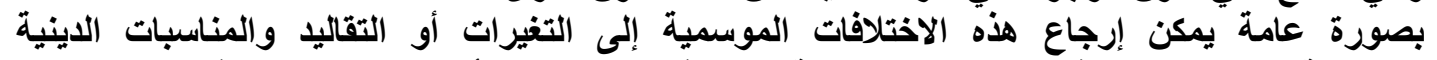

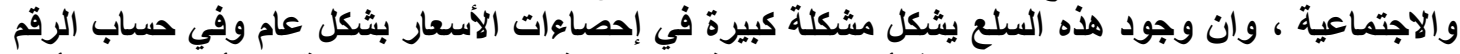

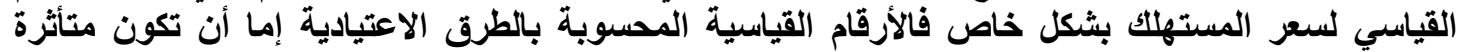

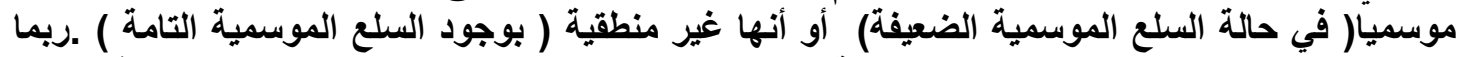

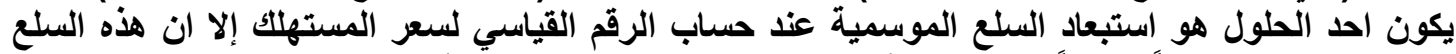

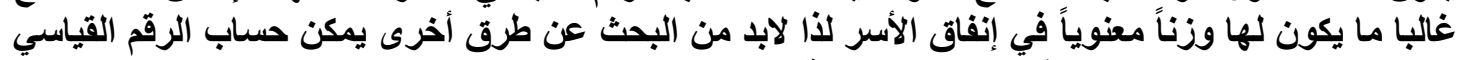

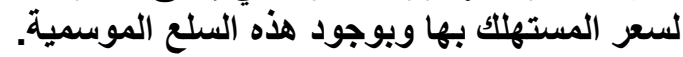

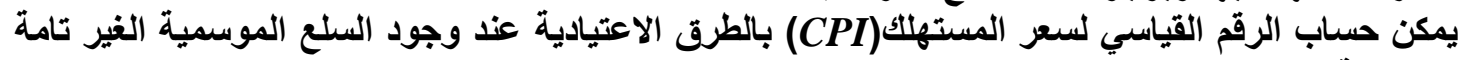

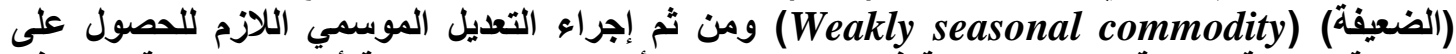

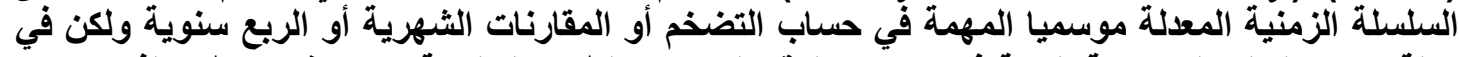

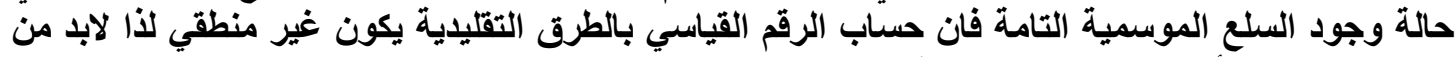

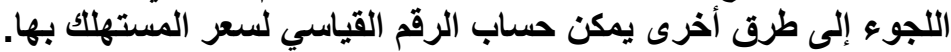

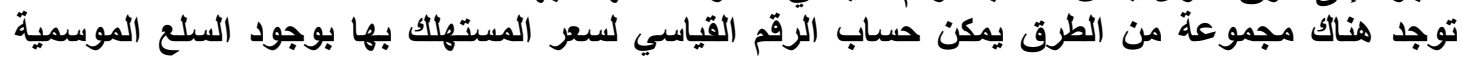

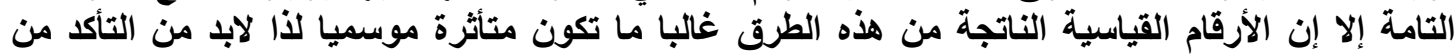

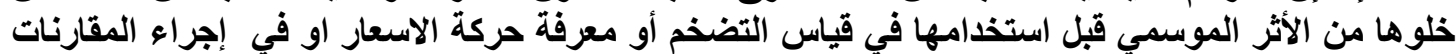

يهاف البحث لوضع بعض الحلول المناسبة لمشكلة وجود السلع الموسمية التامة عند حساب المباب الرقم

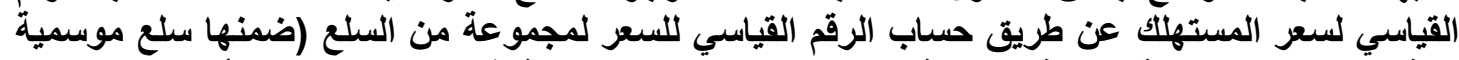

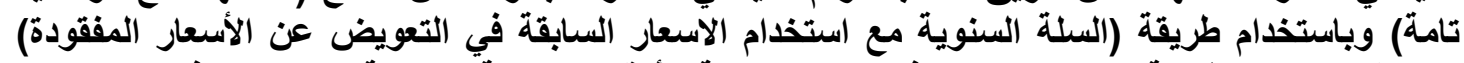

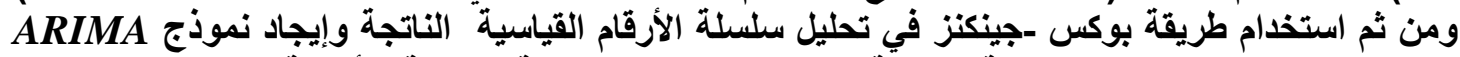

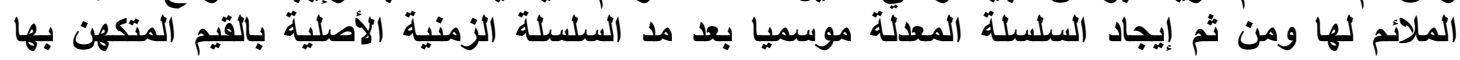




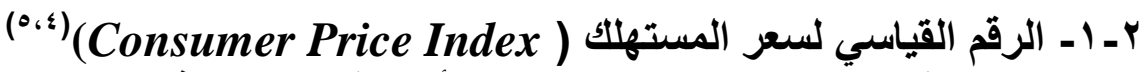

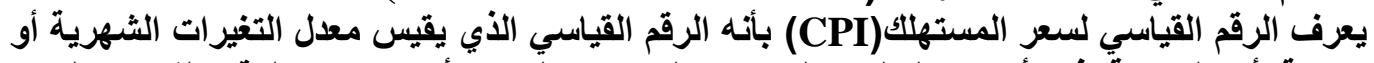

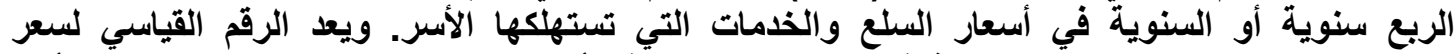

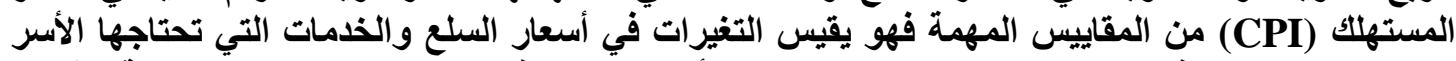

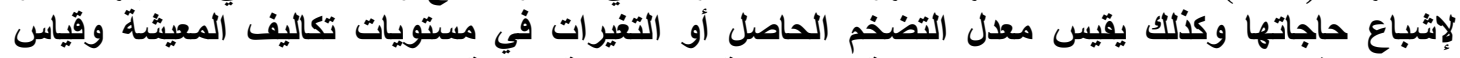

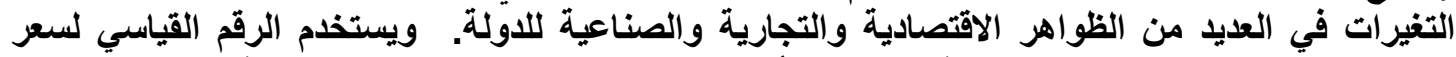

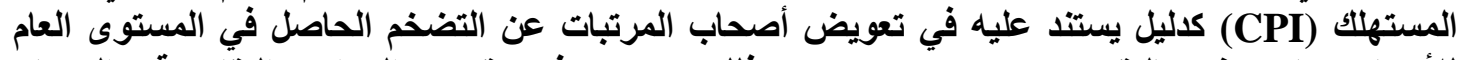

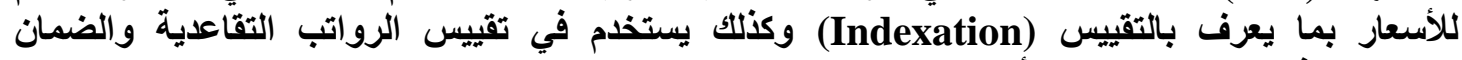
الاجتماعي فضلار عن استعمالات أخرى متعددة.

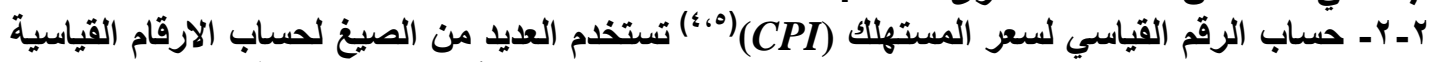

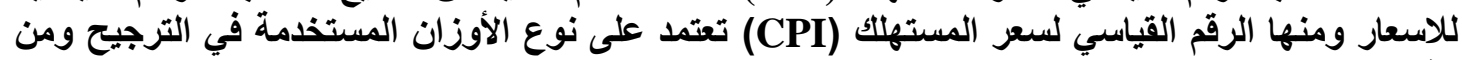

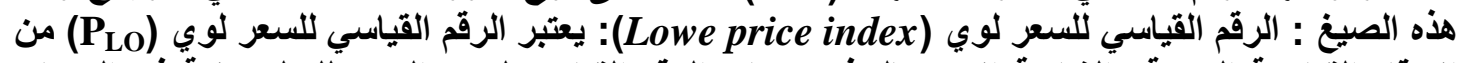

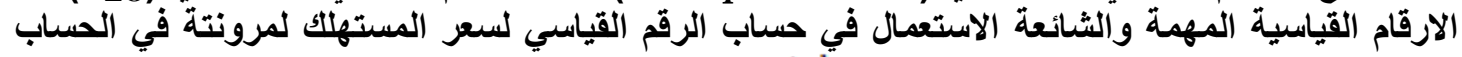

$$
\text { والصيغة العامة لهذا الرقم هي : (Y.1) }
$$

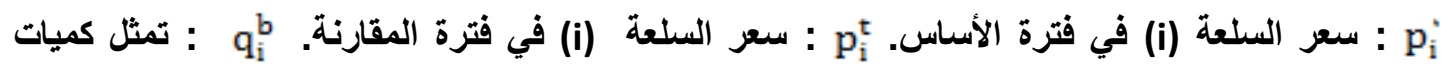

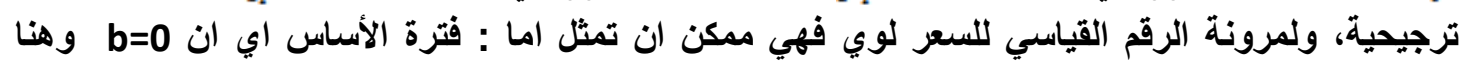

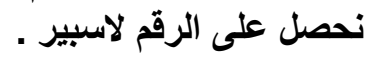

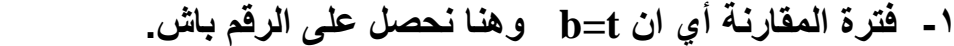

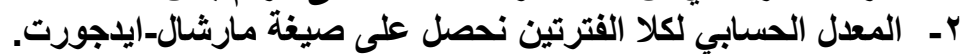

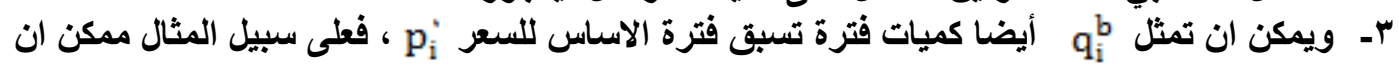

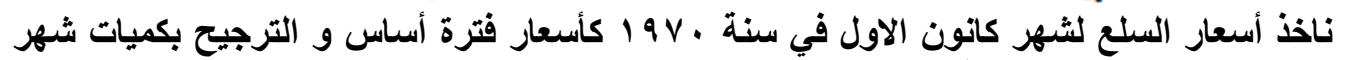

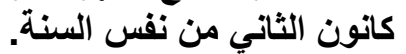
يطلق على متجه الكميات بوزن فترة المصدر في حين يعرف متجه أسعار الأساس بسعر فترة

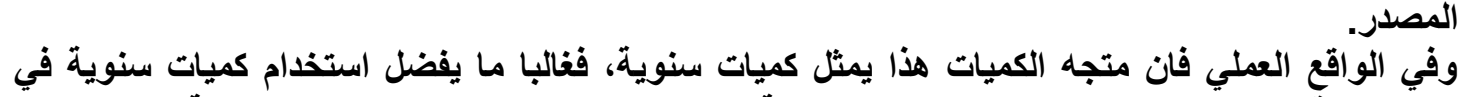

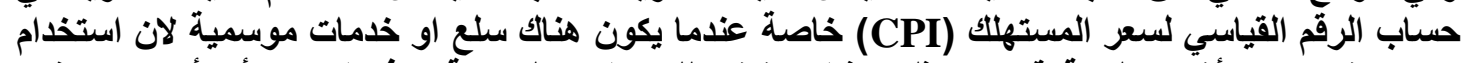

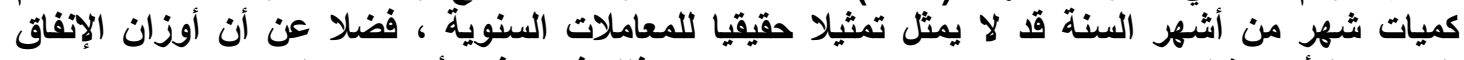

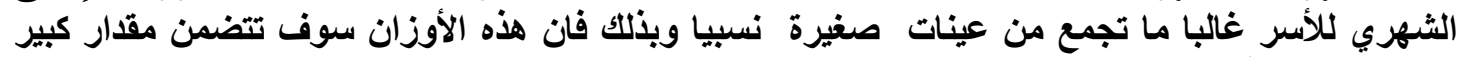




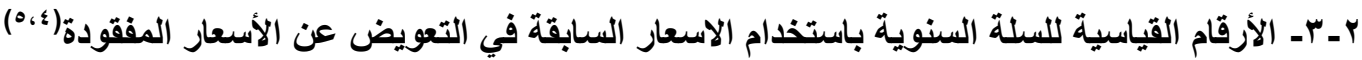
Annual Basket With Carry Forward Prices في عام ب . . ب قدم ايرون ديورت (Erwin Diewert) مجموعة من الطرق للتعامل مع مشكلة السلع

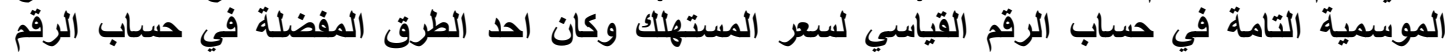

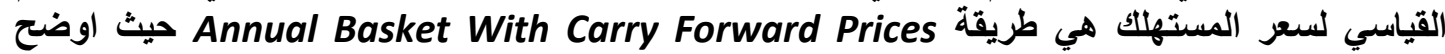

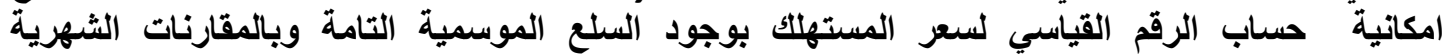

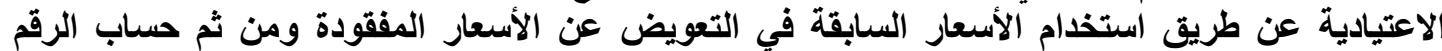

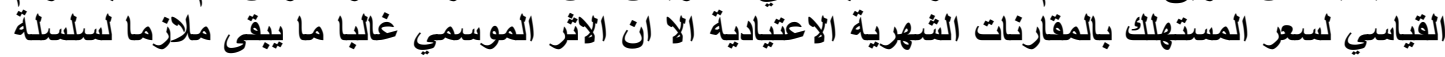

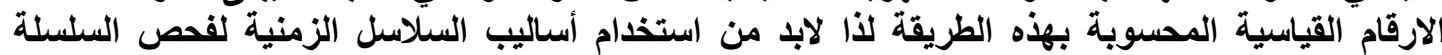

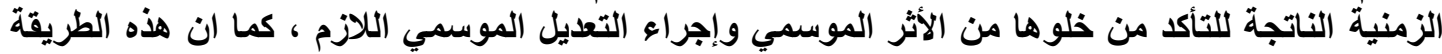

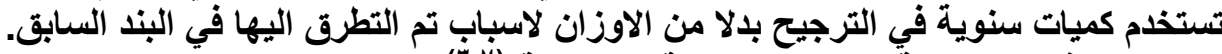
Stochastic Models for seasonal time series

ان أكثر السلاسل الزمنية الاقتصادية تحتوي على مركبة الموسمية التي تظهر في السلسلة بثكل

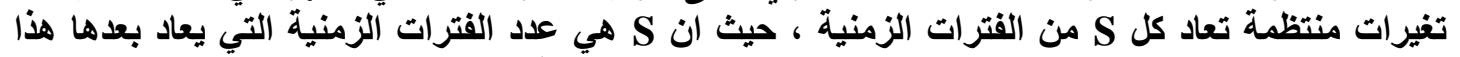

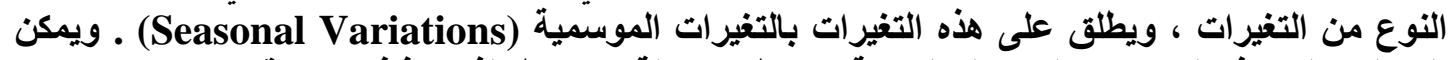

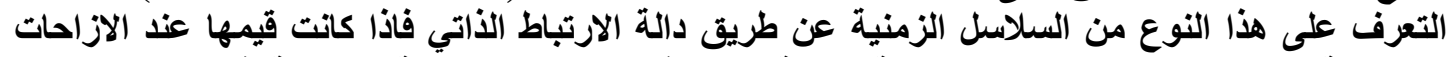

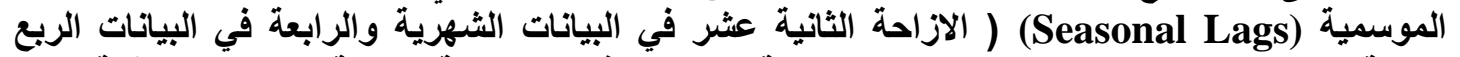

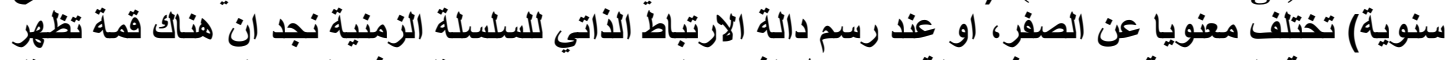

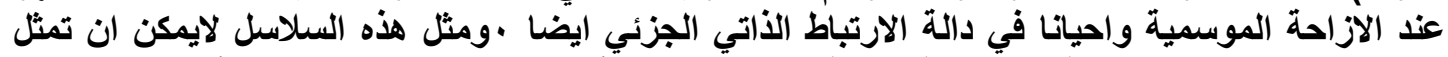

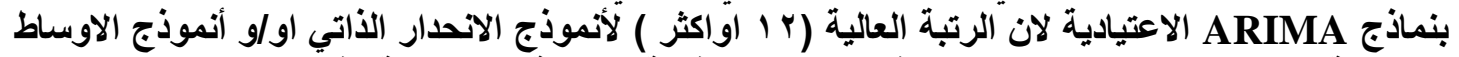

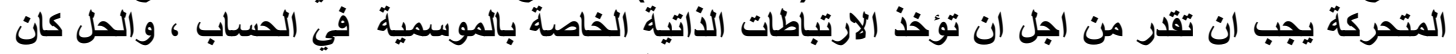

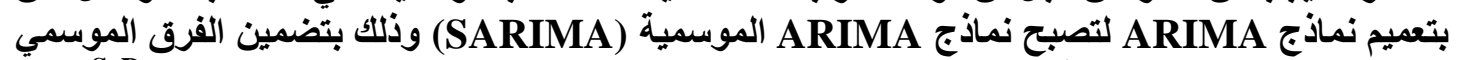
المتمثل بعامل الفروق الخلفية الموسمي( (1-B ${ }^{\text {(1) }}$

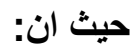

في البيانات الشهرية

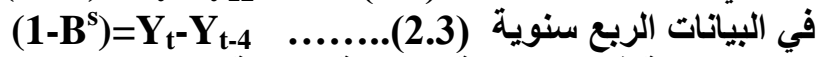

D :Dثل عدد الفروق الموسمية المطبقة لتحقيق الاستقرارية في الموسمية للسلسلة الزمنية.

$\operatorname{ARIMA}(p, d, q)(P, D, Q)_{S}$

ويرمز للنموذج (SARIMA) بالرمز:

حيث ان : هي رتبة انموذج الاتحدار الذاتي غير الموسمي، وان d تمثل الفرق الغير موسمي، q هي رتبة انموذج المتوسطات المتحركة الغير موسمي .

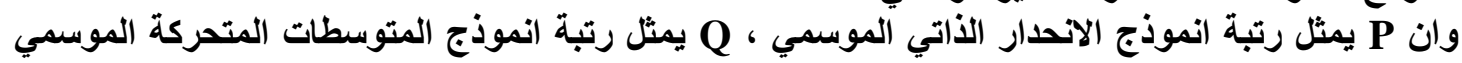

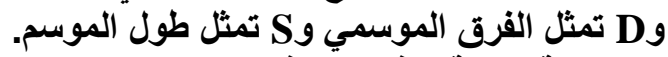

$a_{p}(B) a_{p}\left(B^{s}\right) \nabla^{d} \nabla_{S}^{D} Y_{t}=C+b_{q}(B) b_{Q}\left(B^{s}\right) u_{t} \ldots(\Upsilon, \varepsilon)$ و الصيغة العامة لهذا الأموذج هي : 


$$
\text { (2.8) } \nabla_{\mathrm{S}}=\left(1-B^{S}\right)
$$

r-ه- بعض الاختبارات المستخدمة في تحديا ملائمة الأنموذج لبيانات السلسلة الزمنية:

في هذا الاختبار يتم اختبار مجموعة من الارتباطات الذاتية للاخطاء مرة واحدة وحسب الصيغة التالية : $Q_{B P}=(n-d-S D) \sum_{i=1}^{k} r_{i}^{\uparrow}(u) \ldots($ Ү.q)

و بمقارنـة

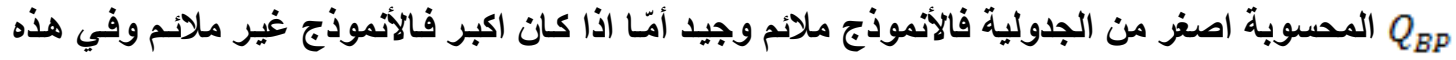

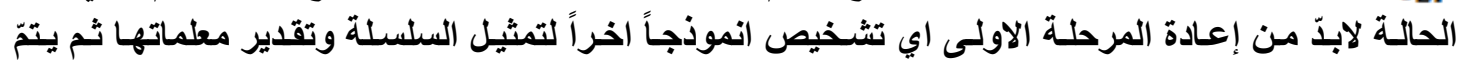

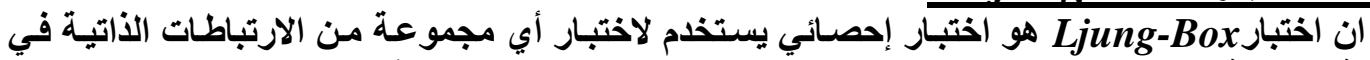

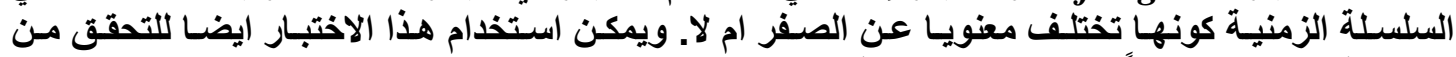

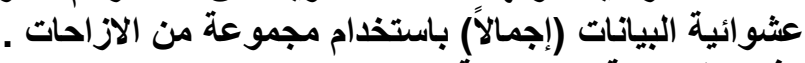

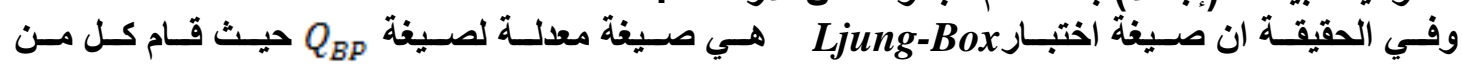
(Box \& بتعديل صيغة اختبار (Ljung,G.M\&Box,G.E.P)

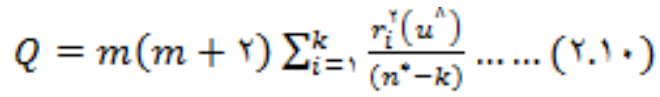

$$
\text { m=(n-d-SD) }
$$

وقد اثبتت تجارب المحاكاة ان اختبارLjung-Box هو الأفضل في العينات الصغيرة والكبيرة. 


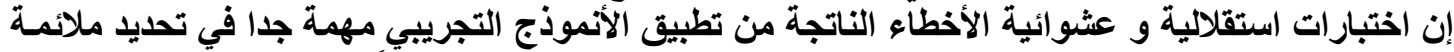

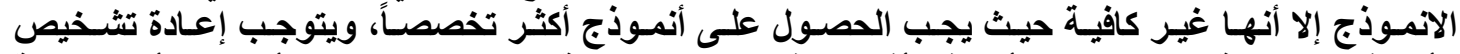

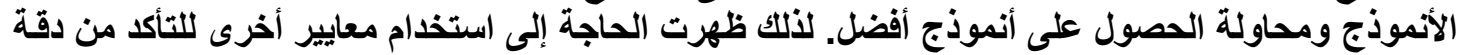

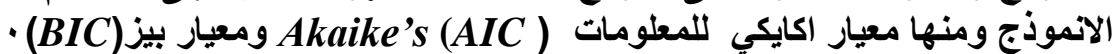

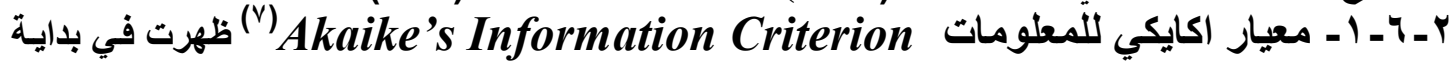

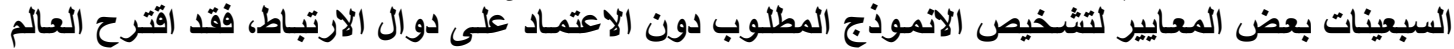

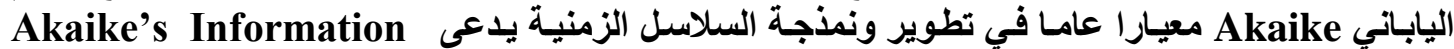
Criterion (AIC)

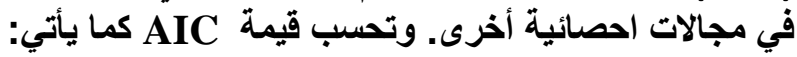

$\operatorname{AIC}(v)=2 \ln \left(\sigma_{u}^{\curlyvee}\right)+2 v \ldots \ldots .(2.11)$

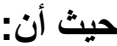

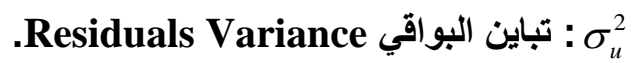
v : عدد معلمات الانموذج المقدرة.

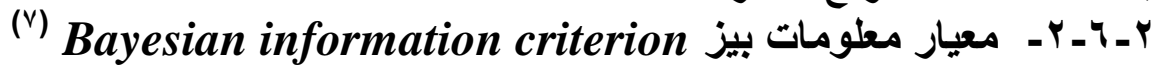
ومن المعايير المهمة ايضا في تثخيص الانموذج المطلوب هو معيار بيز (BIC) حيث قام العالم بيز بتطوير $B I C(v)=2 \operatorname{Ln}\left(\sigma_{u}^{2}\right)+v \operatorname{Ln}(n)$ المعيار السابق(AIC) ليصبح بالصورة الاتية:

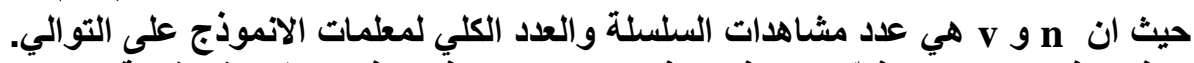

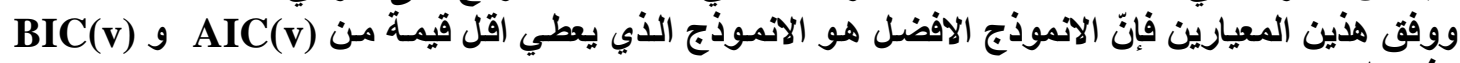

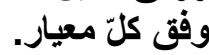

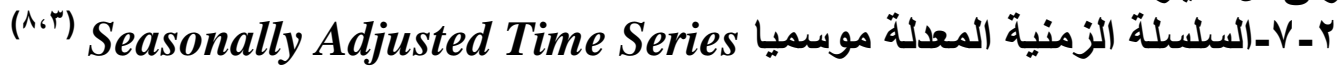

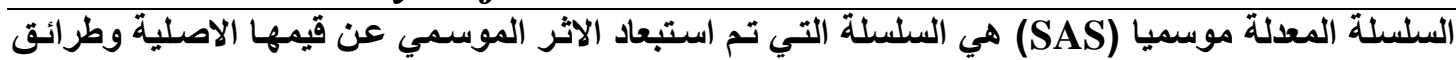

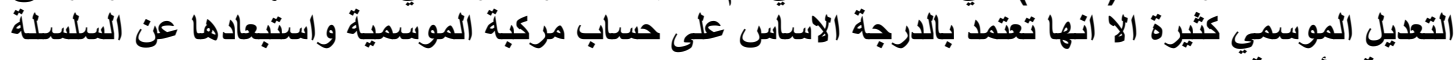

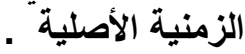

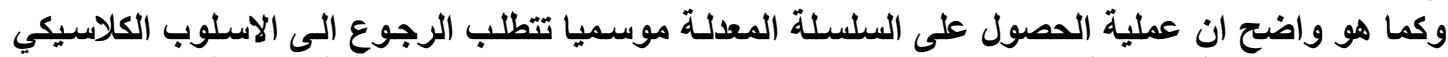

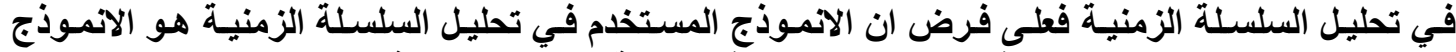

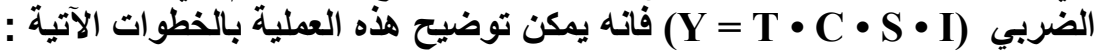

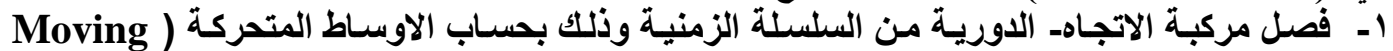

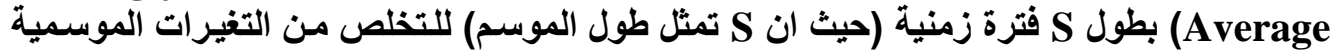

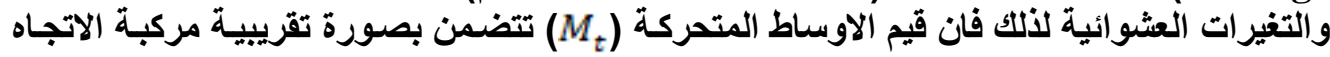

$$
\mathrm{M}_{\mathrm{t}}=\mathrm{T}_{\mathrm{t}} * \mathrm{C}_{\mathrm{t}}
$$

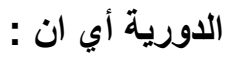

وعندما تكون (S) عددا زوجيا يتم حساب الاوساط المتحركة المركزية (Centered Moving Averages) 


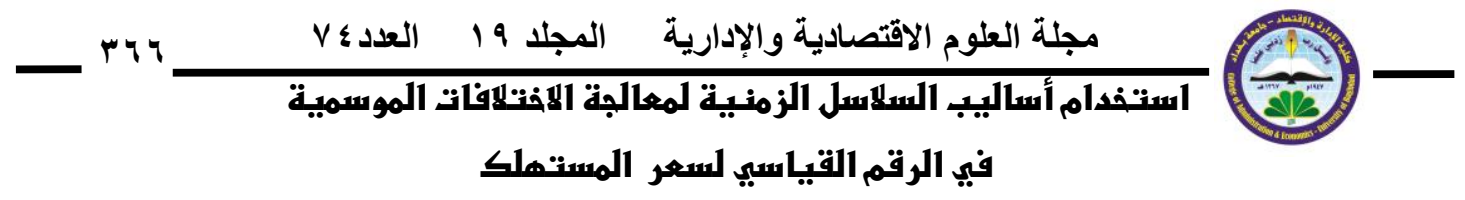

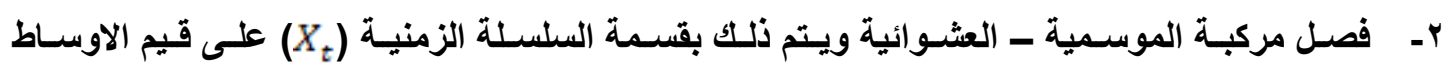
المتحركة (Mt) وضرب الناتج في 100 للحصول على قيم بنسب مئوية أي ان :

$\frac{\mathrm{X}_{\mathrm{t}}}{\mathrm{M}_{\mathrm{t}}}=\frac{\mathrm{S}_{\mathrm{t}} * \mathrm{~T}_{\mathrm{t}} * \mathrm{C}_{\mathrm{t}} * \mathrm{I}_{\mathrm{t}}}{\mathrm{T}_{\mathrm{t}} * \mathrm{C}_{\mathrm{t}}}$

حساب المؤشرات الموسمية (Seasonal Indices) والتي تعد مقياسـا للتغيرات الموسمية الموجودة في التئي

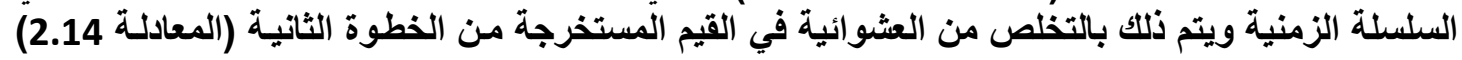
وذللك باستخدام المعدل الوسيط (Median Average Method) . وللحصول على المعدل الون الوسيط يتم ترتيب

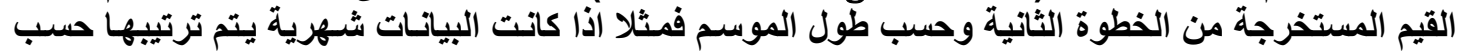

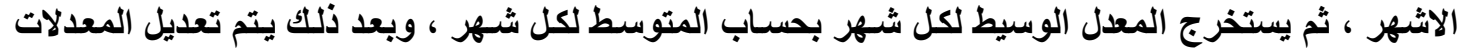

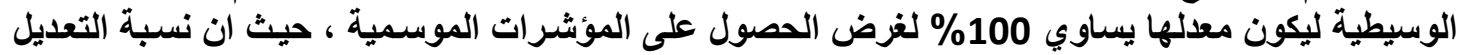
$\mathrm{R}=\frac{1, * * \mathrm{~S}}{\text { Total of Median Averages }}$

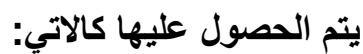

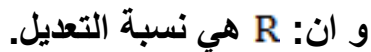

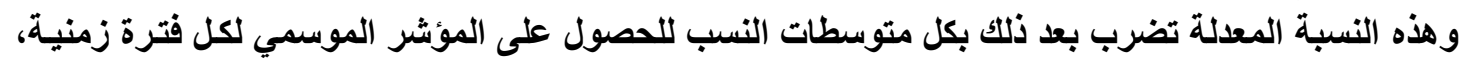

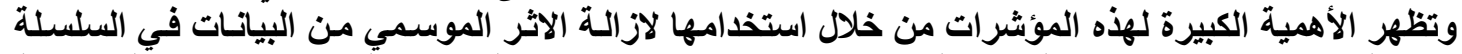

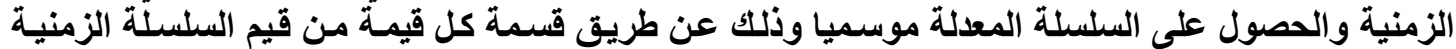

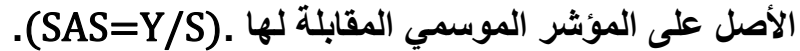
امـا عند استخدام الأنموذج الجمعي (Y= T + C + S + I) في تحليل السلسلة الزمنية فـان السلسلة

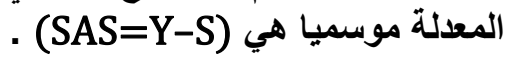


من اجل وضع بعض الحلول لحساب الرقم القياسي لسعر المستهلت (CPI) بوجود السلع الموسمية

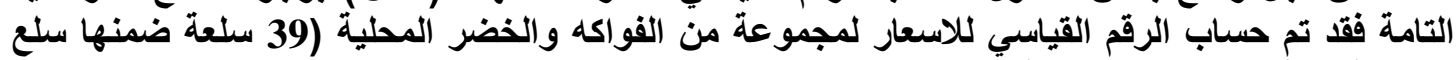

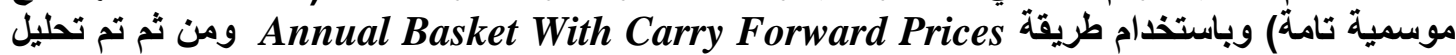
السلسلة الزمنية الناتجة وايجاد الانموذج الملائم لها كما تم ايجاد السلسلة المعلية موسميا بعد اضافة المافية القيم

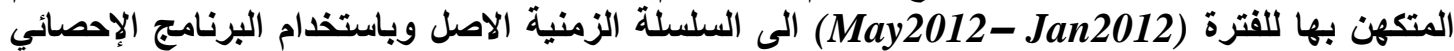
الجاهز (SPSS-19) بعد اجراء التعديلات اللازمة من حيث شكل الدالة المطبقة لحساب الارقام القياسية.

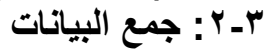
تم اعتماد سجلات وزارة التخطيط الجهاز المركزي للإحصاء في الحصول على البيانات المستخدمة في

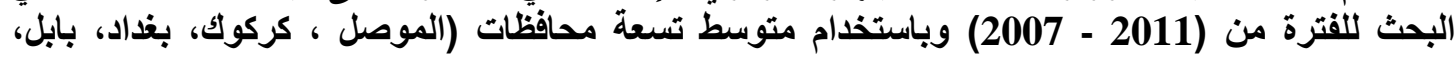
كربلاء، قادسية، مثنى، ميسان، البصرة) لتمثيل بيانات العراق والنتائج موضحة فئنة في الجداول (1,2011)

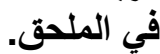

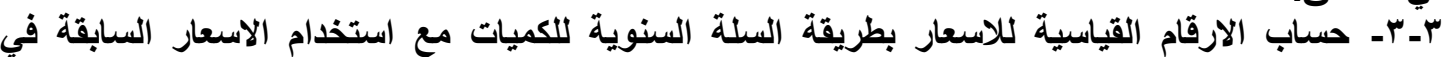

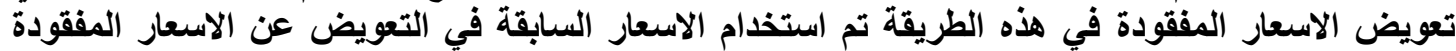

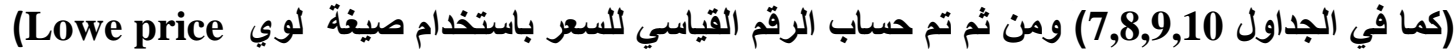

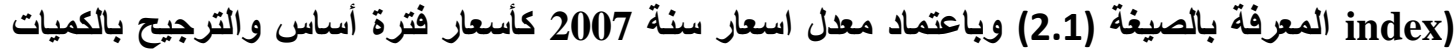

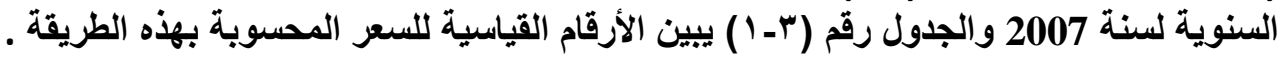

جدول رقم (r-B.W.C.F.P) (1) الارقام القياسية المحسوبة بطريقة)

\begin{tabular}{|c|c|c|c|c|}
\hline DATE & 2008 & 2009 & 2010 & 2011 \\
\hline JAN & 1.06023 & 1.3023 & 1.3907 & 1.5836 \\
\hline FEB & 1.05798 & 1.30025 & 1.45783 & 1.61622 \\
\hline MAR & 1.05662 & 1.29133 & 1.41849 & 1.59025 \\
\hline APR & 1.14324 & 1.42416 & 1.56828 & 1.70993 \\
\hline MAY & 1.54023 & 1.71452 & 1.86141 & 1.98348 \\
\hline JUN & 1.21397 & 1.27813 & 1.38193 & 1.44851 \\
\hline JUL & 1.2274 & 1.28223 & 1.4221 & 1.47293 \\
\hline AUG & 1.29994 & 1.33796 & 1.5724 & 1.6243 \\
\hline SEP & 1.32054 & 1.40489 & 1.57668 & 1.63871 \\
\hline OCT & 1.42419 & 1.67167 & 1.86318 & 1.93027 \\
\hline NOV & 1.30135 & 1.49829 & 1.62056 & 1.74688 \\
\hline DEC & 1.29264 & 1.34819 & 1.57864 & 1.62426 \\
\hline
\end{tabular}


$-r$

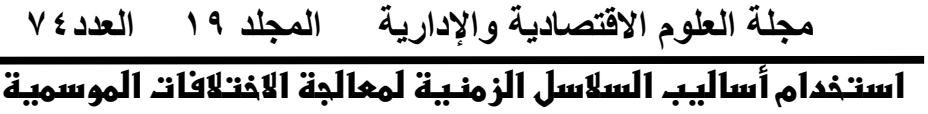

في الرقم القباسبي لستهر المستنهلك

r- ــ ـ تحليل السلسلة الزمنية للأرقام القياسية المحسوبة بطريقة Annual Basket With Carry Forward Prices

ومن اجل تحليل السلسلة الزمنية للارقام القياسية المحسوبة بهذه الطريقة فقد تم اولا رسم السلسلة الزمنية

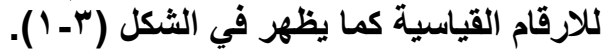
شكل (r- إم اليبين رسم السلسلة الزمنية للارقام القياسية للسعر المحسوبة بطريقة (A.B.W.C.F.P)

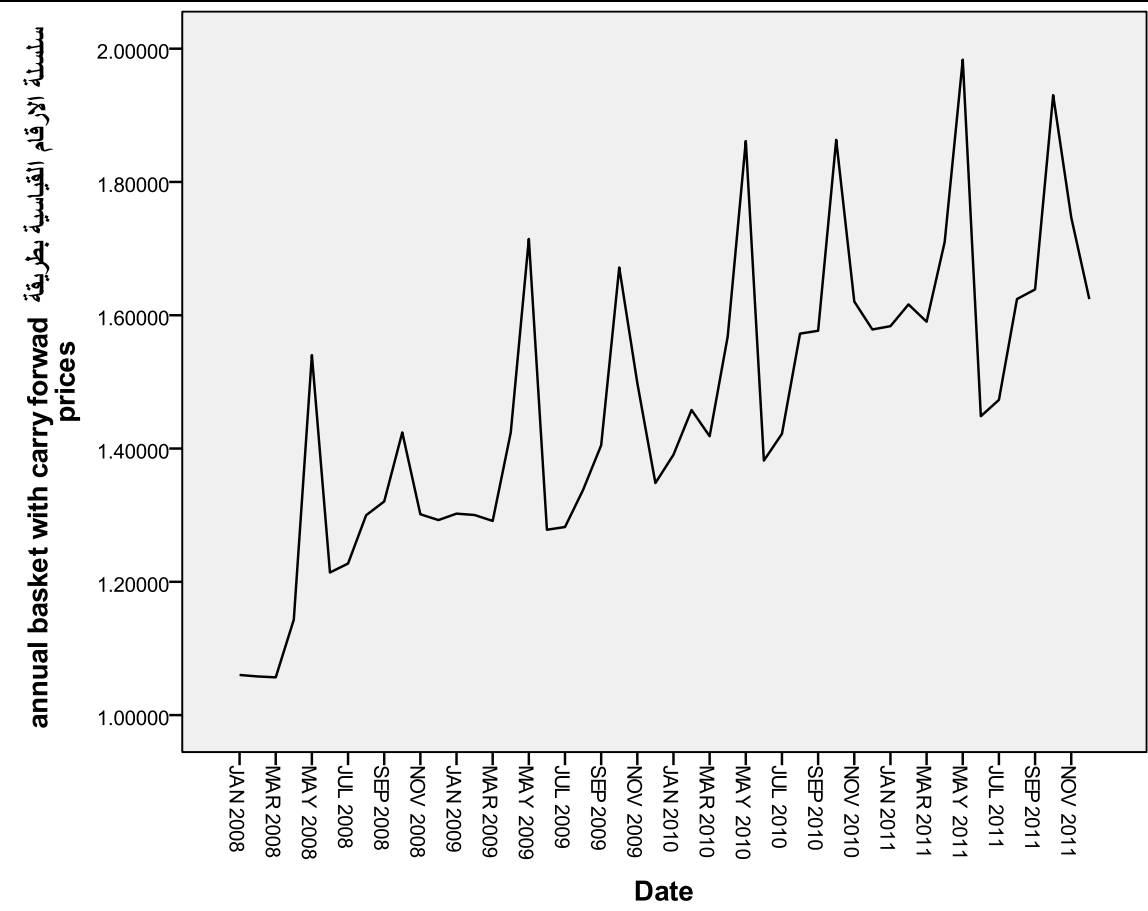

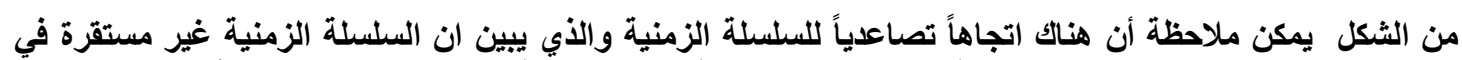

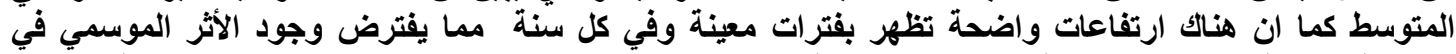

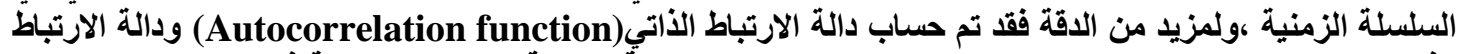

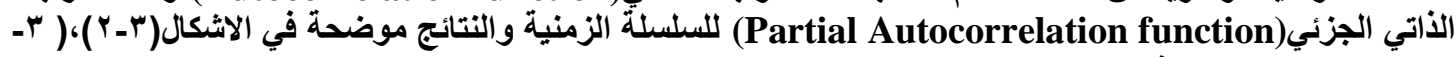
شكل رقم(r-r ب دالة الارتباط الذاتي

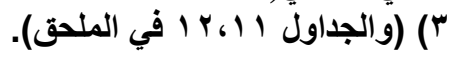

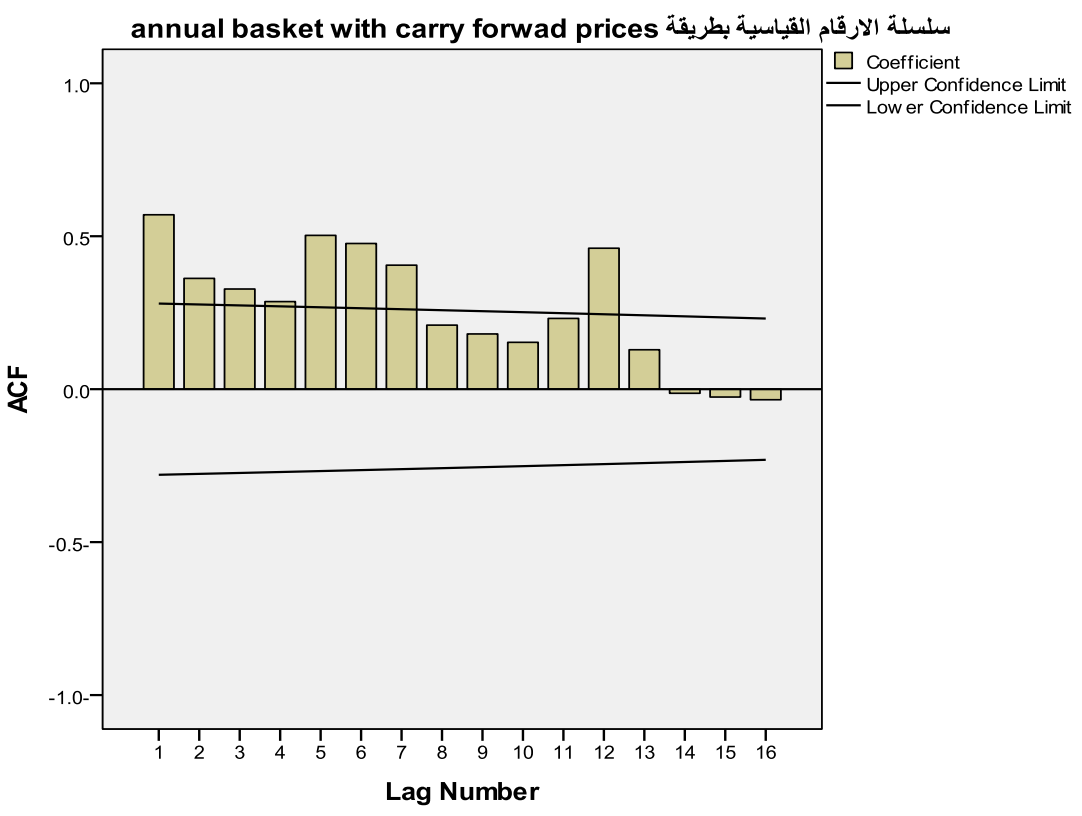


في الرقم القباسبي لسعر المستنهلك

شكل رقم(r-r) دالة الارتباط الذاتي الجزئي

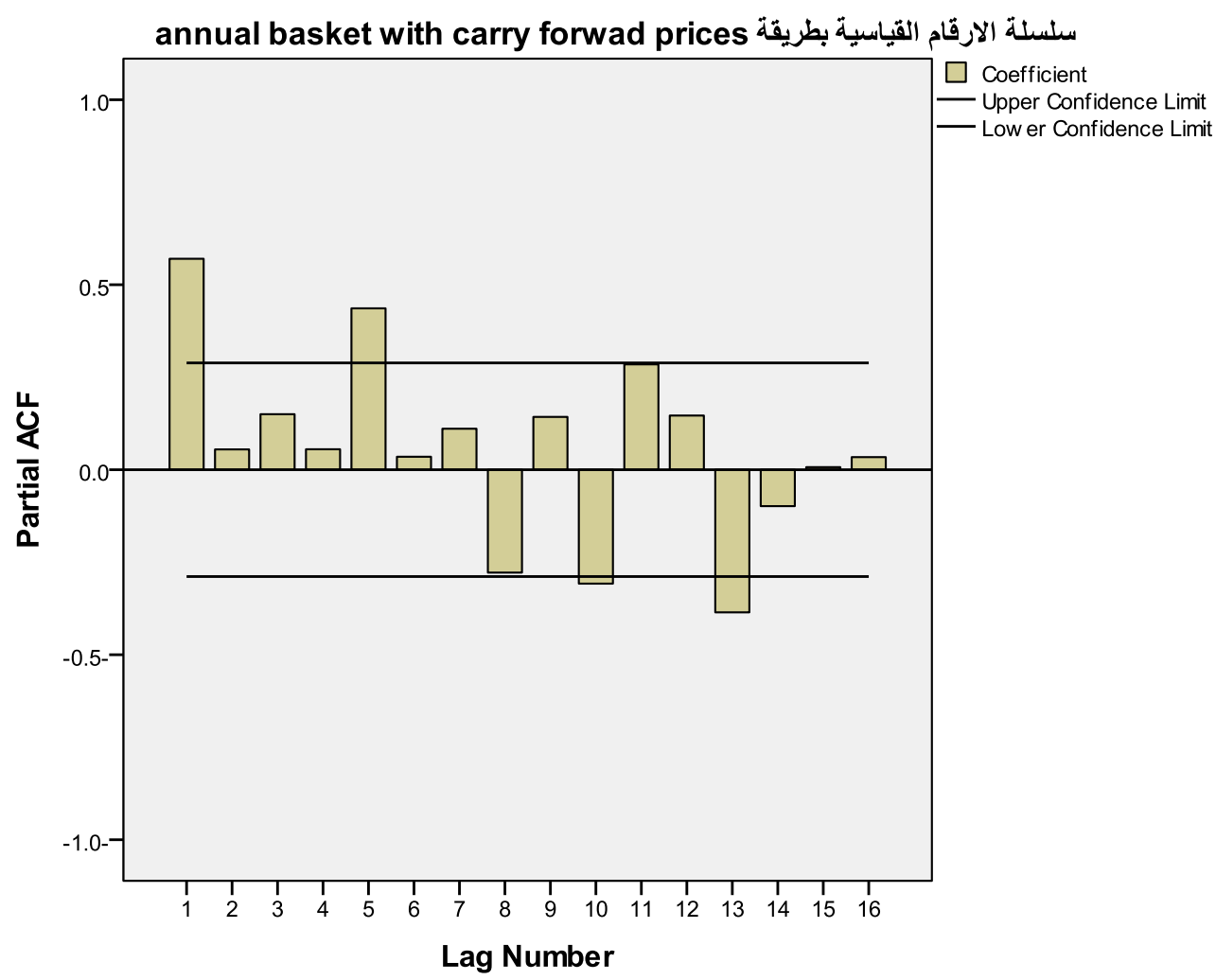

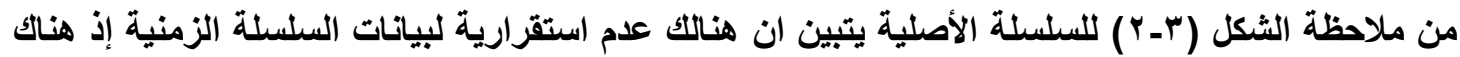

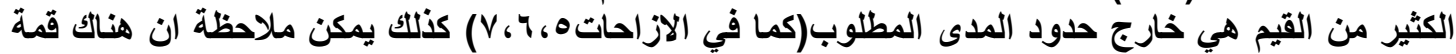

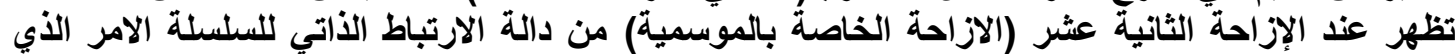

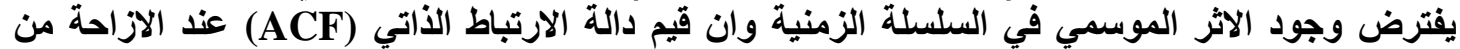

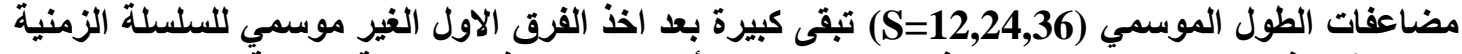

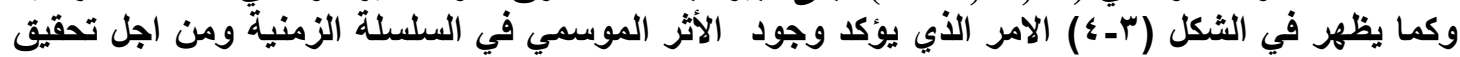

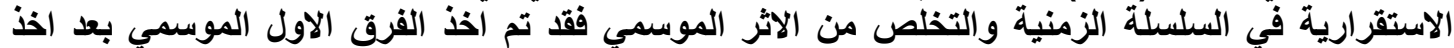

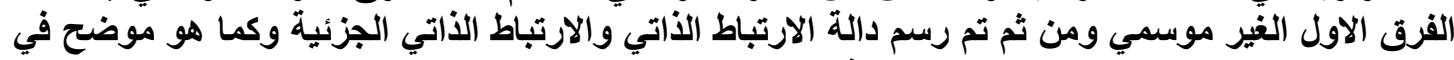

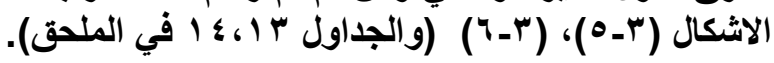


$r v$.

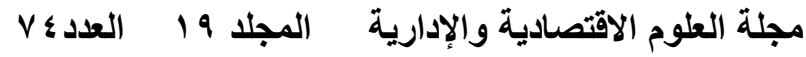

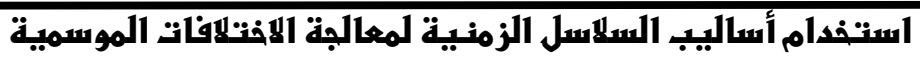
في الرقم القياسي لسعر المستتهلك

شكل (r- ـ ) يبين دالة الارتباط الذاتي للسلسلة الزمنية بعد اخذ الفرق الغير موسمي الاول

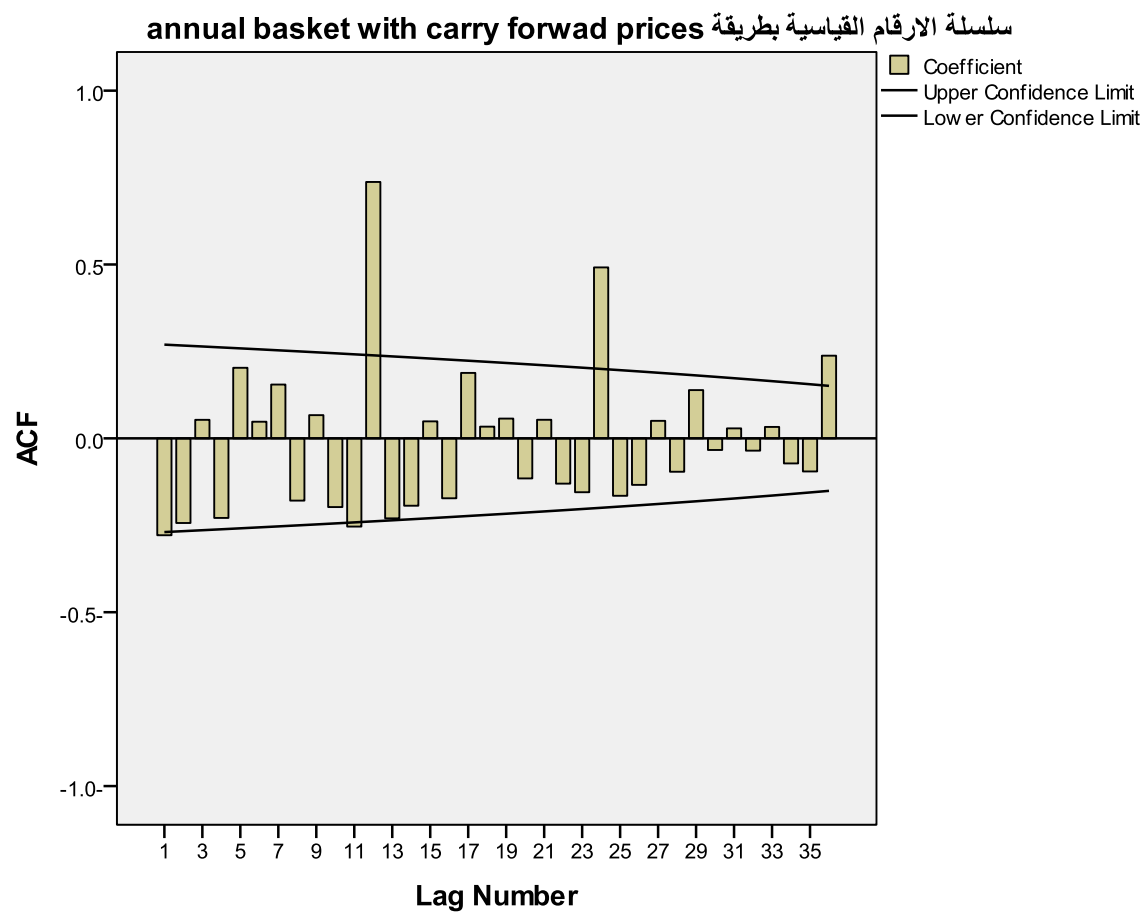

Lag Number

شكل رقم(rــ) يوضح دالة الارتباط الذاتي لسلسلة الارقام القياسية بعد اخذ الفرق الاول الغير موسمي والفرق الاول الموسمي الآمامياتي

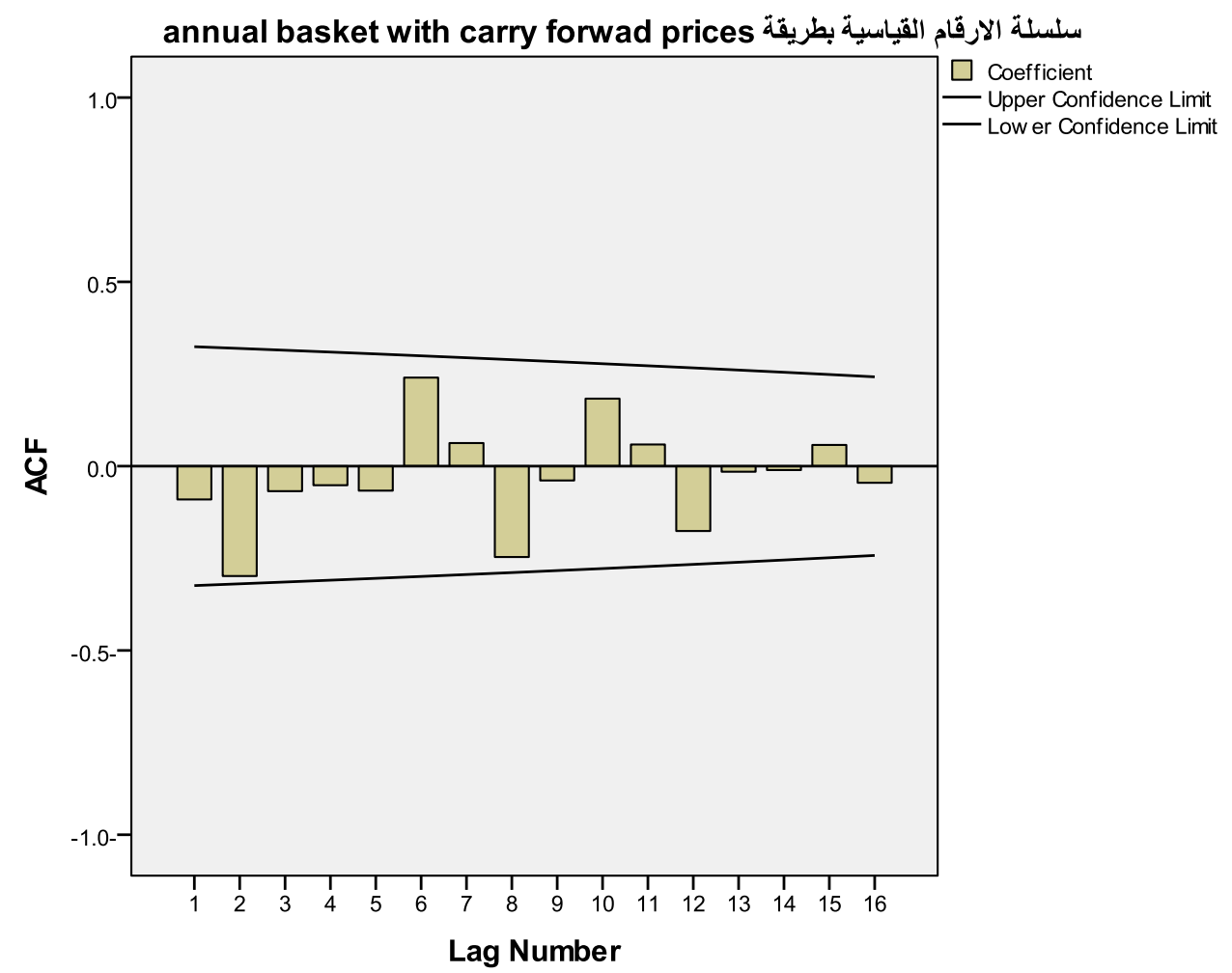


يوضح دالة الارتباط الذاتي الجزئي لسلسلة الارقام القياسية بعد اخذ الفرق الاول الغير موسمي والفرق الاول

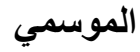

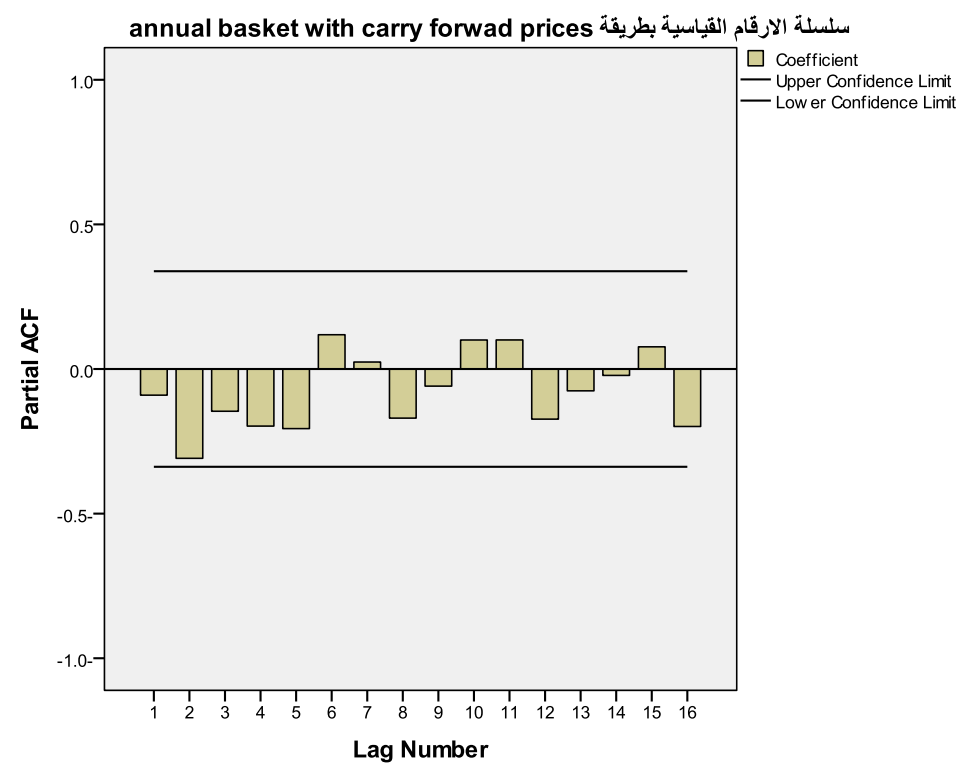

عن طريق الثكل (r-ه) يتبين أنّ جميع قيم دالة الارتباط الذاتي ACF هي في حدود المدى المطلوب وبذلك

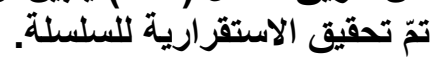

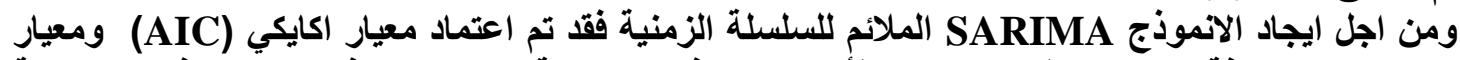

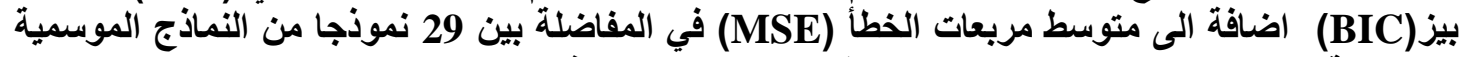
المضاعفة ARIMA(p,d,q)(P,D,Q) وقد تم اختيار الانموذج ARIMA(0,1,1) (0,1,1)

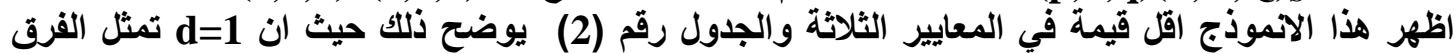

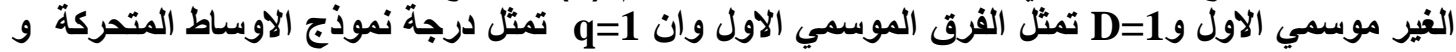

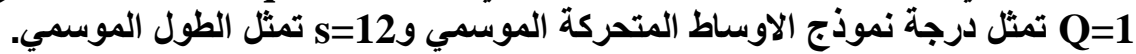

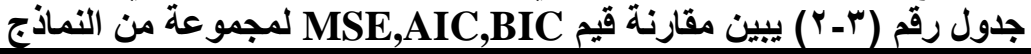

\begin{tabular}{|c|c|c|c|c|}
\hline & Model & MSE & AIC & BIC \\
\hline 1 & ARIMA(0,1,1)(0,1,1) & 0.0039808 & -7.0525439 & -3.3101419 \\
\hline 2 & ARIMA $(1,1,0)(1,1,0)$ & 0.0040233 & -7.0313095 & -3.2889075 \\
\hline 3 & ARIMA(0,1,2)(0,1,1) & 0.0034354 & -5.3472235 & 0.2663795 \\
\hline 4 & ARIMA(1,1,1)(1,1,0) & 0.0034858 & -5.3181238 & 0.2954792 \\
\hline 5 & ARIMA $(2,1,0)(1,1,0)$ & 0.0037709 & -5.1608975 & 0.4527055 \\
\hline 6 & ARIMA(0,1,1)(0,1,2) & 0.0040106 & -5.0376396 & 0.5759634 \\
\hline 7 & ARIMA $(1,1,0)(1,1,1)$ & 0.0041009 & -4.9931141 & 0.6204889 \\
\hline 8 & ARIMA $(1,1,0)(2,1,0)$ & 0.0041043 & -4.9914221 & 0.622181 \\
\hline 9 & ARIMA(0,1,2)(0,1,2) & 0.003426 & -3.3527464 & 4.1320576 \\
\hline 10 & ARIMA(2,1,1)(1,1,0) & 0.0034582 & -3.3339891 & 4.150815 \\
\hline 11 & ARIMA(0,1,3)(0,1,1) & 0.0034735 & -3.3252054 & 4.1595986 \\
\hline
\end{tabular}


في الرقم القياسي لسعر المستهلك

\begin{tabular}{|c|c|c|c|c|}
\hline التسلس||c| & Model & MSE & AIC & BIC \\
\hline 12 & ARIMA(1,1,1)(1,1,1) & 0.0035365 & -3.2892413 & 4.1955628 \\
\hline 13 & ARIMA(1,1,1)(2,1,0) & 0.0035398 & -3.2873488 & 4.1974552 \\
\hline 14 & ARIMA(3,1,0)(1,1,0) & 0.0038221 & -3.1339014 & 4.3509026 \\
\hline 15 & ARIMA(2,1,0)(1,1,1) & 0.0038451 & -3.1219208 & 4.3628833 \\
\hline 16 & ARIMA(2,1,0)(2,1,0) & 0.0038454 & -3.1217541 & 4.36305 \\
\hline 17 & ARIMA(0,1,3)(0,1,2) & 0.00351 & -1.304281 & 8.051724 \\
\hline 18 & ARIMA(2,1,1)(1,1,1) & 0.0035215 & -1.2977578 & 8.0582473 \\
\hline 19 & ARIMA(2,1,1)(2,1,0) & 0.0035398 & -1.287379 & 8.068626 \\
\hline 20 & ARIMA(2,1,2)(1,1,0) & 0.0035582 & -1.2770177 & 8.0789873 \\
\hline 21 & ARIMA(3,1,0)(2,1,0) & 0.003892 & -1.0976434 & 8.2583616 \\
\hline 22 & ARIMA(3,1,0)(1,1,1) & 0.003896 & -1.0955945 & 8.2604106 \\
\hline 23 & ARIMA(3,1,1)(1,1,0) & 0.003566 & 0.7273918 & 11.954598 \\
\hline 24 & ARIMA(2,1,2)(1,1,1) & 0.0036352 & 0.7657934 & 11.993 \\
\hline 25 & ARIMA(3,1,1)(1,1,1) & 0.0036471 & 0.772332 & 11.999538 \\
\hline 26 & ARIMA(3,1,2)(1,1,0) & 0.0036894 & 0.7954126 & 12.022619 \\
\hline 27 & ARIMA(3,1,2)(1,1,1) & 0.0037757 & 2.8416599 & 15.940067 \\
\hline 28 & ARIMA(3,1,3)(1,1,0) & 0.0038003 & 2.8546662 & 15.953073 \\
\hline 29 & ARIMA(3,1,3)(1,1,1) & 0.0038816 & 4.8970007 & 19.866609 \\
\hline & & & & \\
& & &
\end{tabular}

وبعد اختيار الانموذج التجريبي ARIMA(0,1,1) (0,1,1) تم تقدير معلمات الانموذج واختبار معنوية

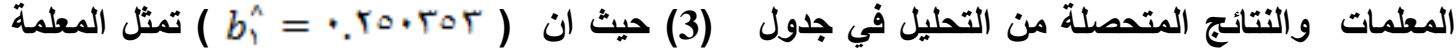

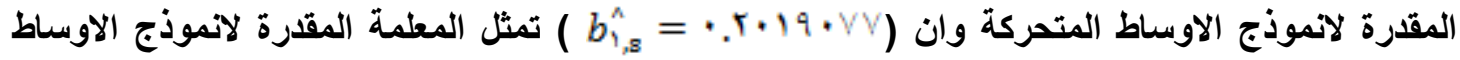
المتحركة الموسمي وان ( 0.0045312 - C C تمثل ثابت الانموذج ،علما ان قيمة معامل التحديد

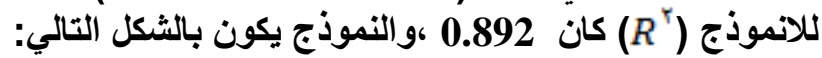
$(1-B)\left(1-B^{12}\right) Y_{t}=(1+(0.2503) B)\left(1+(0.2019) B^{12}\right) u_{t}+(-0.00453)$

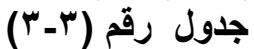

\begin{tabular}{|c|c|c|c|c|}
\hline & Estimate & SE & t & Sig. \\
\hline Constant & $\mathbf{- 0 . 0 0 4 5 3 1 2}$ & $\mathbf{0 . 0 0 7 0 0 4 4}$ & $\mathbf{- 0 . 6 4 6 9 0 3 7}$ & $\mathbf{0 . 5 2 2 3 0 7 9}$ \\
\hline Difference & 1 & & & \\
\hline MA & $\mathbf{0 . 2 5 0 3 5 3}$ & $\mathbf{0 . 1 7 2 5 4 7 1}$ & 1.4509254 & $\mathbf{0 . 1 5 6 5 3 3 7}$ \\
\hline Seasonal Difference & 1 & & & \\
\hline MA, Seasonal & $\mathbf{0 . 2 0 1 9 0 7 7}$ & $\mathbf{0 . 1 9 4 3 6 0 1}$ & 1.038833 & $\mathbf{0 . 3 0 6 6 6 8 5}$ \\
\hline
\end{tabular}




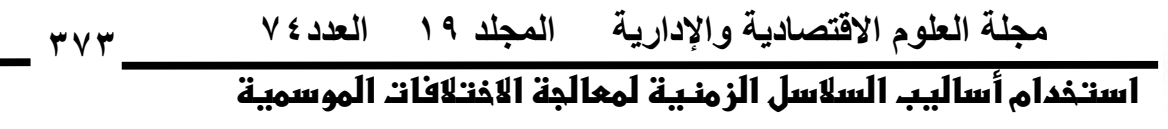

\section{في الرقم القباستي لستعر المستنهلك}

ومن اجل التحقق من ملائمة الانموذج تم اختبار مجموعة من الارتباطات الأتية للاخطاء مرة واحدة وذلك

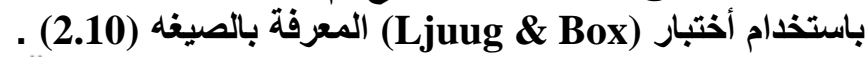

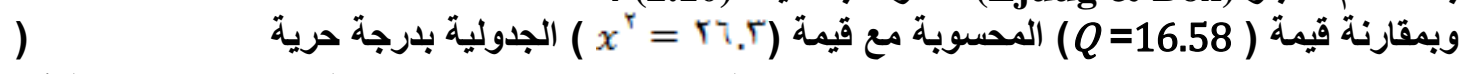

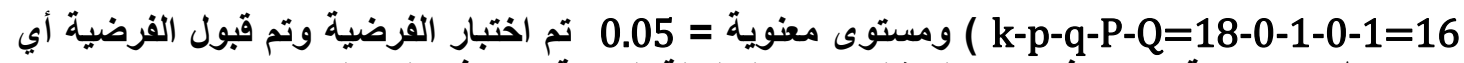

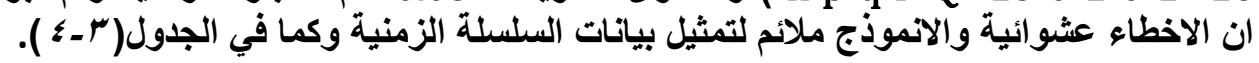

جدول رقم(r- ؛) يبين اختبار (Ljuug \& Box) للانموذج

\begin{tabular}{|c|c|c|}
\hline \multirow[t]{2}{*}{ Residual ACF } & $\begin{array}{c}\text { Ljung-Box } \\
Q(18)\end{array}$ & \\
\hline & Statistics & DF \\
\hline 0.080136216 & 16.58003436 & 16 \\
\hline-0.292698896 & & \\
\hline-0.170102582 & & \\
\hline-0.159792166 & & \\
\hline-0.028048247 & & \\
\hline 0.301471894 & & \\
\hline 0.04101412 & & \\
\hline-0.275605178 & & \\
\hline-0.09042609 & & \\
\hline 0.118722934 & & \\
\hline 0.068348541 & & \\
\hline 0.030307759 & & \\
\hline-0.005715156 & & \\
\hline-0.088582788 & & \\
\hline-0.001326951 & & \\
\hline-0.072532127 & & \\
\hline-0.022077398 & & \\
\hline 0.088870663 & & \\
\hline
\end{tabular}

بعد التاكد من ملائمة الانموذج للبيانات ولغرض المقارنة وضعت النتائج للقيم التقديرية مع الحقيقية

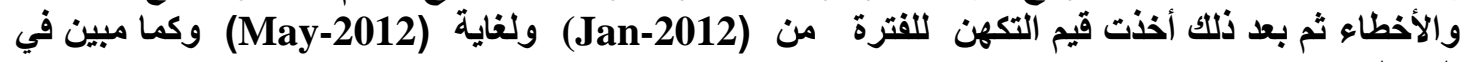

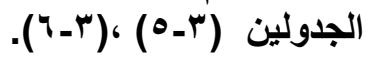




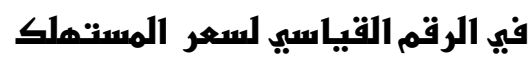

جدول (r- ) يبين قيم السلسلة الاصل مع القيم المتنبأ بها والاخطاء

\begin{tabular}{|c|c|c|c|}
\hline Date & Origin Data & Predicted Data & Residual \\
\hline Jan-08 & 1.06023 & & \\
\hline Feb-08 & 1.05798 & & \\
\hline Mar-08 & 1.05662 & & \\
\hline Apr-08 & 1.14324 & & \\
\hline May-08 & 1.54023 & & \\
\hline Jun-08 & 1.21397 & & \\
\hline Jul-08 & 1.2274 & & \\
\hline Aug-08 & 1.29994 & & \\
\hline Sep-08 & 1.32054 & & \\
\hline Oct-08 & 1.42419 & & \\
\hline Nov-08 & 1.30135 & & \\
\hline Dec-08 & 1.29264 & & \\
\hline Jan-09 & 1.3023 & & \\
\hline Feb-09 & 1.30025 & 1.29552 & 0.00473 \\
\hline Mar-09 & 1.29133 & 1.29324 & $-.00191-$ \\
\hline Apr-09 & 1.42416 & 1.3739 & 0.05025 \\
\hline May-09 & 1.71452 & 1.80404 & $-.08952-$ \\
\hline Jun-09 & 1.27813 & 1.40613 & $-.12800-$ \\
\hline Jul-09 & 1.28223 & 1.31908 & $-.03685-$ \\
\hline Aug-09 & 1.33796 & 1.35946 & $-.02150-$ \\
\hline Sep-09 & 1.40489 & 1.35941 & 0.04548 \\
\hline Oct-09 & 1.67167 & 1.49262 & 0.17905 \\
\hline Nov-09 & 1.49829 & 1.49947 & $-.00118-$ \\
\hline Dec-09 & 1.34819 & 1.48534 & $-.13715-$ \\
\hline Jan-10 & 1.3907 & 1.38791 & 0.00279 \\
\hline Feb-10 & 1.45783 & 1.38253 & 0.07531 \\
\hline Mar-10 & 1.41849 & 1.42616 & $-.00767-$ \\
\hline Apr-10 & 1.56828 & 1.53886 & 0.02942 \\
\hline May-10 & 1.86141 & 1.86655 & $-.00515-$ \\
\hline Jun-10 & 1.38193 & 1.44226 & $-.06032-$ \\
\hline Jul-10 & 1.4221 & 1.39754 & 0.02457 \\
\hline Aug-10 & 1.5724 & 1.46953 & 0.10287 \\
\hline Sep-10 & 1.57668 & 1.59918 & $-.02250-$ \\
\hline Oct-10 & 1.86318 & 1.81204 & 0.05115 \\
\hline Nov-10 & 1.62056 & 1.68139 & $-.06083-$ \\
\hline Dec-10 & 1.57864 & 1.50771 & 0.07092 \\
\hline Jan-11 & 1.5836 & 1.59179 & $-.00819-$ \\
\hline Feb-11 & 1.61622 & 1.6332 & $-.01699-$ \\
\hline Mar-11 & 1.59025 & 1.58193 & 0.00832 \\
\hline Apr-11 & 1.70993 & 1.72711 & $-.01718-$ \\
\hline May-11 & 1.98348 & 2.00535 & $-.02187-$ \\
\hline Jun-11 & 1.44851 & 1.51685 & $-.06834-$ \\
\hline Jul-11 & 1.47293 & 1.49326 & $-.02033-$ \\
\hline Aug-11 & 1.6243 & 1.60429 & 0.02001 \\
\hline Sep-11 & 1.63871 & 1.62875 & 0.00996 \\
\hline Oct-11 & 1.93027 & 1.90675 & 0.02352 \\
\hline Nov-11 & 1.74688 & 1.69208 & 0.0548 \\
\hline Dec-11 & 1.62426 & 1.66933 & $-.04508-$ \\
\hline
\end{tabular}


rro

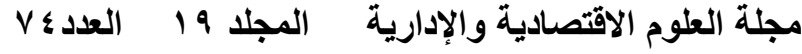

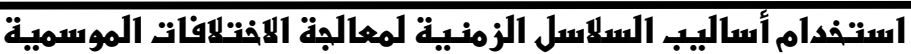

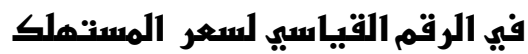

جدول رقم (بـ ؟) يبين القيم المقدرة باستخدام الانموذج المقدر للاشهر الخمسة الاولى من سنة 2012

\begin{tabular}{|c|c|c|c|c|c|c|}
\hline $\begin{array}{l}\text { Model } \\
\text { ARIMA(0,1,1 }\end{array}$ & 1,1) & Jan-12 & Feb-12 & Mar-12 & Apr-12 & May-12 \\
\hline سلسلة الأرقام & Forecast & 1.6412043 & 1.6723085 & 1.6392721 & 1.7583097 & 2.0308711 \\
\hline القياسية بطريقة & UCL & 1.7680658 & 1.8308568 & 1.8241539 & 1.9662159 & 2.2594947 \\
\hline $\begin{array}{l}\text { with carry } \\
\text { forward } \\
\text { prices }\end{array}$ & LCL & 1.5143429 & 1.5137602 & 1.4543903 & 1.5504034 & 1.8022476 \\
\hline & & & & & & \\
\hline & & & & & & \\
\hline
\end{tabular}

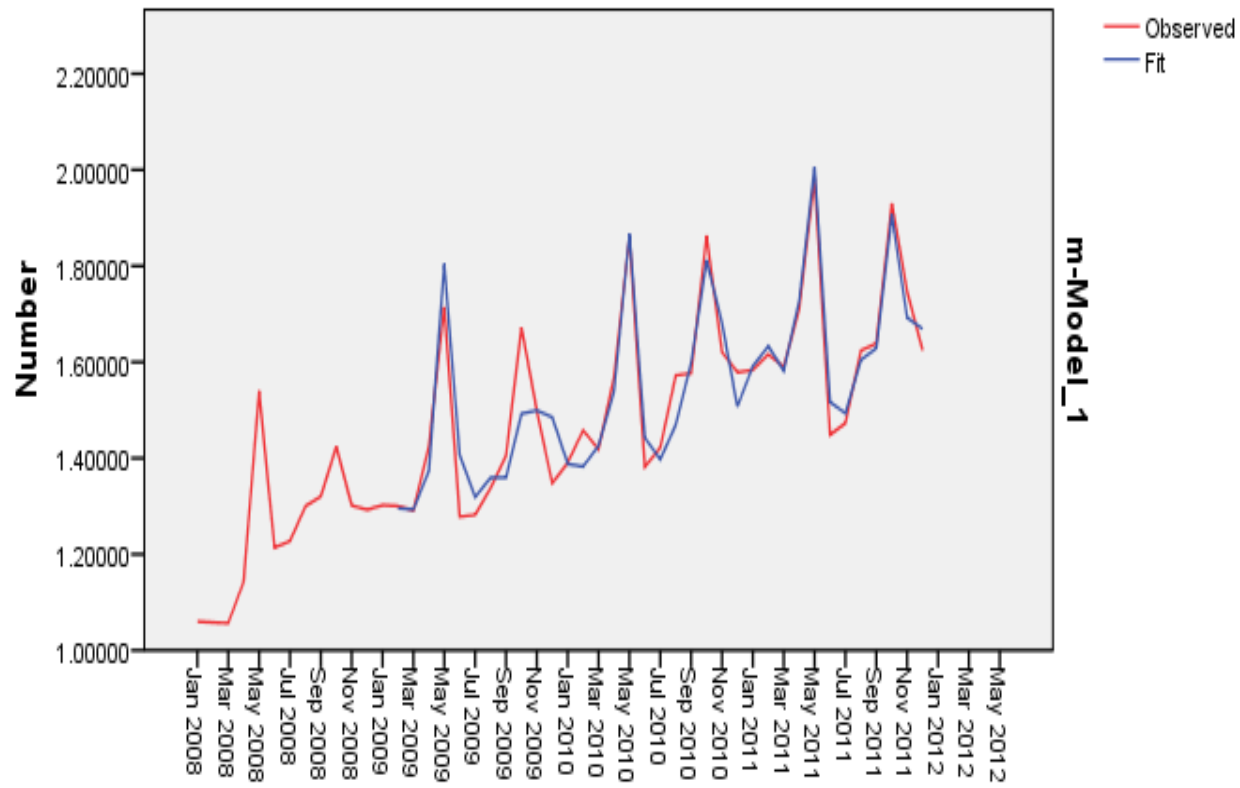

Date

(V-r) يمثل السلسلة الزمنية الاصلية مع القيم المتنبأ بها

شكل

r_- ايجاد السلسلة المعدلة موسميا (SAS) لسلسلة الارقام القياسية المحسوبة بطريقة Annual Basket With Carry Forward Prices

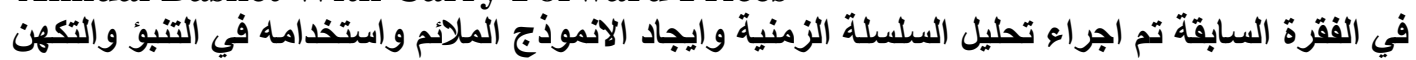

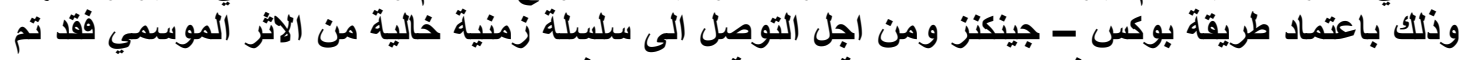

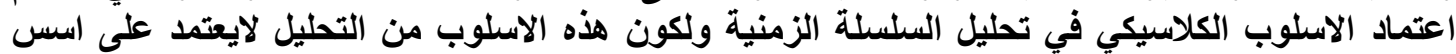

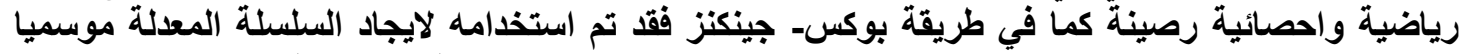
فقط. تم اعتماد الانموذج الضربي (

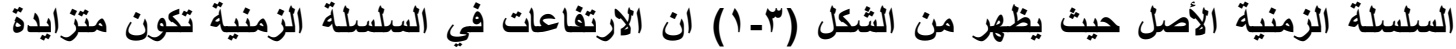

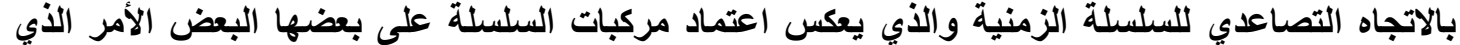

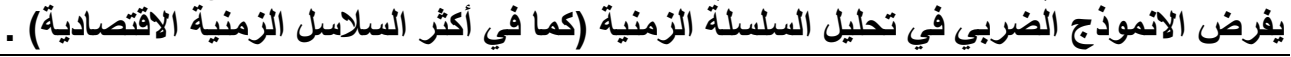


والجدول رقم (Y-r) يبين ذلك ،حيث ان العمود الثاني يبين قيم السلسلة الزمنية الأصل (بعد مدها بالقيم

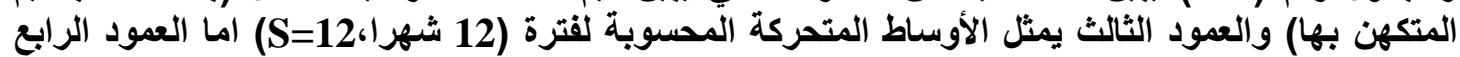

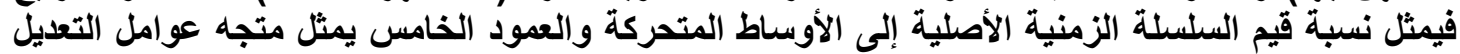

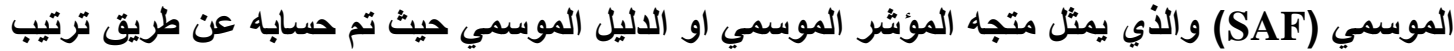

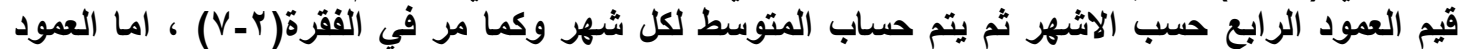

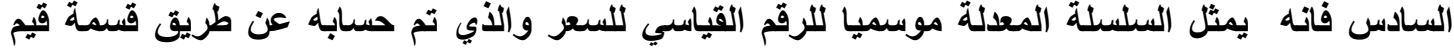
السلسلة الزمنية الأصل على متجه التعديل الموسئ الميل (SAF).

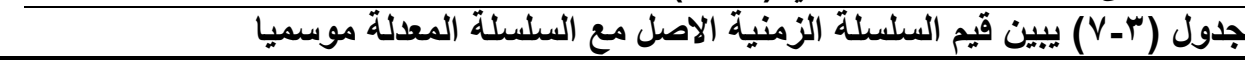

\begin{tabular}{|c|c|c|c|c|c|}
\hline DATE_ & $\begin{array}{l}\text { Original } \\
\text { Series } \\
\end{array}$ & $\begin{array}{l}\text { Moving } \\
\text { Average } \\
\text { Series } \\
\end{array}$ & $\begin{array}{c}\text { Ratio of } \\
\text { Original Series } \\
\text { to Moving } \\
\text { Average Series } \\
(\%) \\
\end{array}$ & $\begin{array}{c}\text { Seasonal Factor } \\
(\%)\end{array}$ & $\begin{array}{c}\text { Seasonally } \\
\text { Adjusted } \\
\text { Series } \\
\end{array}$ \\
\hline Jan-08 & 1.0602318 & . & . & 95.937285 & 1.1051301 \\
\hline Feb-08 & 1.0579844 & . & . & 97.811488 & 1.0816566 \\
\hline Mar-08 & 1.0566184 & . & . & 94.586553 & 1.1170916 \\
\hline Apr-08 & 1.1432436 & . & . & 103.27896 & 1.1069473 \\
\hline May-08 & 1.5402321 & . & . & 120.82248 & 1.2747893 \\
\hline Jun-08 & 1.2139665 & . & . & 89.106807 & 1.3623723 \\
\hline Jul-08 & 1.227404 & 1.2448612 & 98.597664 & 90.615593 & 1.3545175 \\
\hline Aug-08 & 1.2999397 & 1.2650336 & 102.7593 & 97.885729 & 1.3280176 \\
\hline Sep-08 & 1.3205365 & 1.2852224 & 102.74771 & 98.234356 & 1.3442716 \\
\hline Oct-08 & 1.4241909 & 1.3047821 & 109.15163 & 114.84643 & 1.2400829 \\
\hline Nov-08 & 1.3013491 & 1.3281916 & 97.979017 & 101.22626 & 1.2855844 \\
\hline Dec-08 & 1.2926368 & 1.3427154 & 96.270346 & 95.648059 & 1.3514511 \\
\hline Jan-09 & 1.3023007 & 1.3480622 & 96.605388 & 95.937285 & 1.35745 \\
\hline Feb-09 & 1.3002505 & 1.3526309 & 96.127512 & 97.811488 & 1.3293433 \\
\hline Mar-09 & 1.2913343 & 1.3557992 & 95.245249 & 94.586553 & 1.3652409 \\
\hline Apr-09 & 1.4241582 & 1.3628282 & 104.5002 & 103.27896 & 1.3789433 \\
\hline May-09 & 1.7145179 & 1.3834514 & 123.93048 & 120.82248 & 1.4190388 \\
\hline Jun-09 & 1.2781277 & 1.3998631 & 91.303765 & 89.106807 & 1.4343772 \\
\hline Jul-09 & 1.2822289 & 1.4044927 & 91.294803 & 90.615593 & 1.4150201 \\
\hline Aug-09 & 1.3379585 & 1.4118593 & 94.765705 & 97.885729 & 1.3668575 \\
\hline Sep-09 & 1.4048854 & 1.4249913 & 98.589051 & 98.234356 & 1.4301365 \\
\hline Oct-09 & 1.6716689 & 1.4355872 & 116.44495 & 114.84643 & 1.4555689 \\
\hline Nov-09 & 1.4982897 & 1.4475976 & 103.50181 & 101.22626 & 1.4801393 \\
\hline Dec-09 & 1.3481919 & 1.4598383 & 92.352144 & 95.648059 & 1.409534 \\
\hline Jan-10 & 1.3907001 & 1.4684887 & 94.702814 & 95.937285 & 1.4495929 \\
\hline Feb-10 & 1.4578339 & 1.480145 & 98.492643 & 97.811488 & 1.4904526 \\
\hline Mar-10 & 1.4184858 & 1.499682 & 94.585774 & 94.586553 & 1.4996697 \\
\hline
\end{tabular}




\begin{tabular}{|c|c|c|c|c|c|}
\hline Apr-10 & 1.5682825 & 1.5139979 & 103.58552 & 103.27896 & 1.5184919 \\
\hline May-10 & 1.8614057 & 1.5299575 & 121.66389 & 120.82248 & 1.5406121 \\
\hline Jun-10 & 1.381933 & 1.5401471 & 89.727343 & 89.106807 & 1.5508726 \\
\hline Jul-10 & 1.422104 & 1.5593507 & 91.19847 & 90.615593 & 1.5693811 \\
\hline Aug-10 & 1.5724032 & 1.5754255 & 99.808158 & 97.885729 & 1.6063662 \\
\hline Sep-10 & 1.5766755 & 1.5886242 & 99.247861 & 98.234356 & 1.6050144 \\
\hline DATE & $\begin{array}{l}\text { Original } \\
\text { Series }\end{array}$ & $\begin{array}{l}\text { Moving } \\
\text { Average } \\
\text { Series }\end{array}$ & $\begin{array}{c}\text { Ratio of } \\
\text { Original Series } \\
\text { to Moving } \\
\text { Average Series } \\
(\%)\end{array}$ & $\begin{array}{c}\text { Seasonal Factor } \\
(\%)\end{array}$ & $\begin{array}{c}\text { Seasonally } \\
\text { Adjusted } \\
\text { Series }\end{array}$ \\
\hline Oct-10 & 1.8631845 & 1.6029379 & 116.2356 & 114.84643 & 1.6223269 \\
\hline Nov-10 & 1.6205645 & 1.6147418 & 100.36059 & 101.22626 & 1.6009328 \\
\hline Dec-10 & 1.5786357 & 1.6249143 & 97.151939 & 95.648059 & 1.6504629 \\
\hline Jan-11 & 1.5835981 & 1.6304622 & 97.125718 & 95.937285 & 1.6506597 \\
\hline Feb-11 & 1.6162175 & 1.6346973 & 98.869526 & 97.811488 & 1.65238 \\
\hline $\begin{array}{l}\text { Mar-11 } \\
\text { Apr-11 }\end{array}$ & $\begin{array}{l}1.5902507 \\
1.7099294\end{array}$ & $\begin{array}{l}1.6390216 \\
1.6441916\end{array}$ & $\begin{array}{c}97.02439 \\
103.99819\end{array}$ & $\begin{array}{l}94.586553 \\
103.27896\end{array}$ & $\begin{array}{l}1.6812652 \\
1.6556416\end{array}$ \\
\hline May-11 & 1.983475 & 1.6497824 & 120.22646 & 120.82248 & 1.641644 \\
\hline Jun-11 & 1.4485083 & 1.6603088 & 87.243304 & 89.106807 & 1.6255866 \\
\hline Jul-11 & 1.4729255 & 1.6641108 & 88.511263 & 90.615593 & 1.6254658 \\
\hline Aug-11 & 1.624295 & 1.668911 & 97.326644 & 97.885729 & 1.6593788 \\
\hline Sep-11 & 1.6387146 & 1.6735853 & 97.916407 & 98.234356 & 1.6681685 \\
\hline Oct-11 & 1.9302742 & 1.6776703 & 115.05683 & 114.84643 & 1.6807438 \\
\hline Nov-11 & 1.7468819 & 1.681702 & 103.87583 & 101.22626 & 1.72572 \\
\hline Dec-11 & 1.6242593 & 1.6856516 & 96.35795 & 95.648059 & 1.6981623 \\
\hline Jan-12 & 1.6412 & . & . & 95.937285 & 1.7107009 \\
\hline Feb-12 & 1.67231 & . & . & 97.811488 & 1.7097276 \\
\hline Mar-12 & 1.63927 & $\cdot$ & $\cdot$ & 94.586553 & 1.7330899 \\
\hline Apr-12 & 1.75831 & . & . & 103.27896 & 1.7024862 \\
\hline May-12 & 2.03087 & . & . & 120.82248 & 1.6808709 \\
\hline
\end{tabular}


rVA

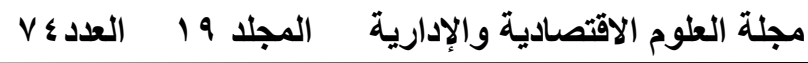

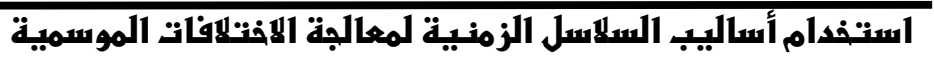
في الرقم القباسبي لسعثر المستنهلك

شكل (r-^) يمثل السلسلة الزمنية الاصل مع السلسلة المعدلة موسميا

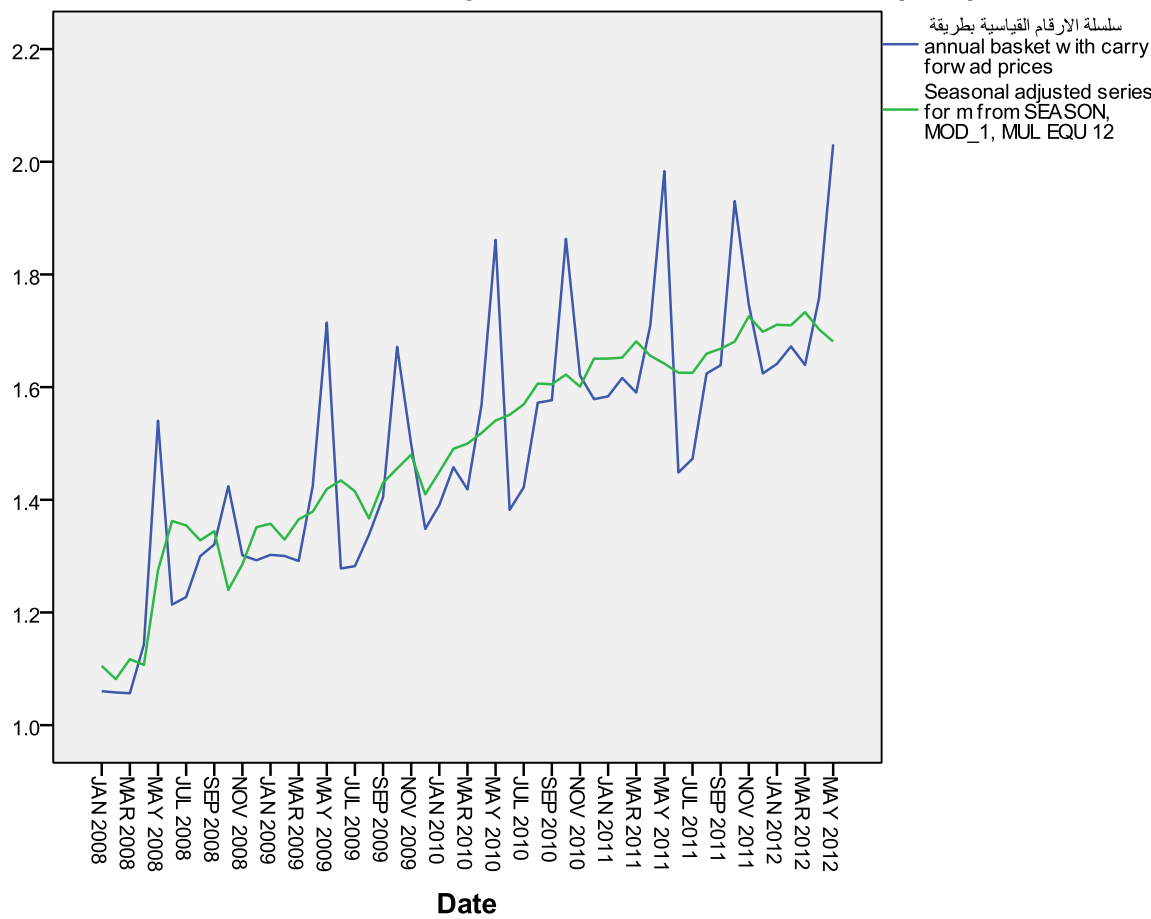


لقد توصل الباحث الى مجموعة من النتائج عن طريق هذه الاراسة النظرية والتطبيقية واهمها هي:

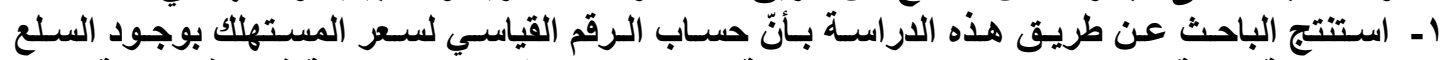

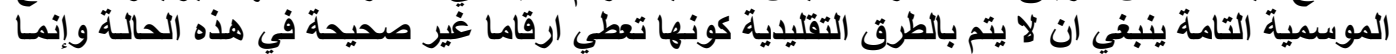

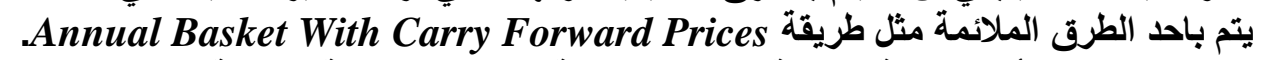

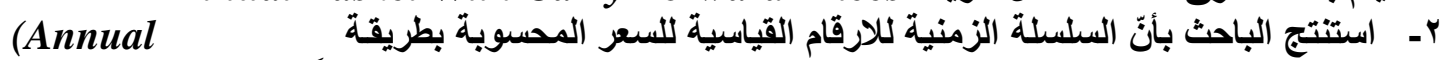
Basket With Carry Forward Prices)

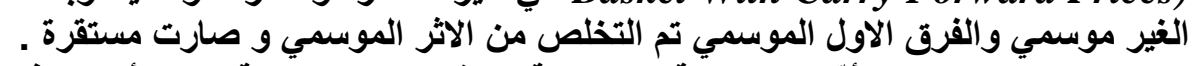

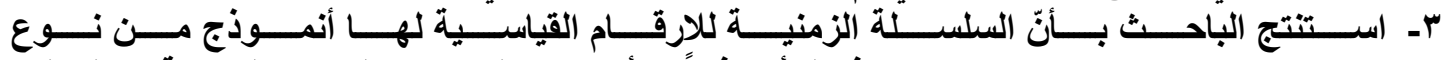

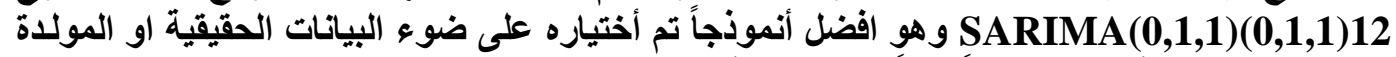
وكان توفيق هذا الأنموذج توفيقاً جيداً بعد تطبيق أختبار (Ljung and Box).

1- الاهتمام بجمع البيانات الخاصة بأسعار وكميات السلع الداخلة في حساب الرقم القياسي لسعر المستهرك.

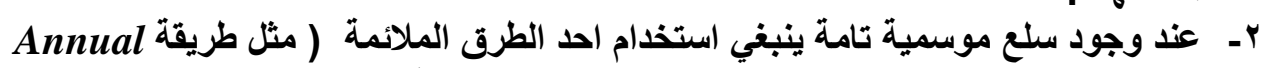
(Basket With Carry Forward Prices

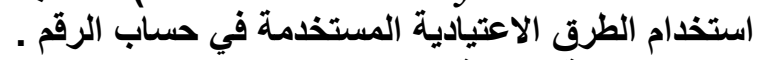

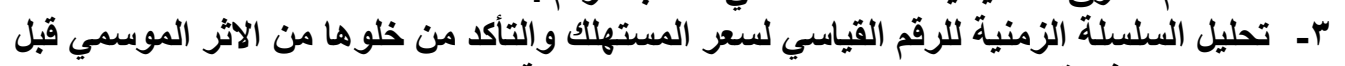

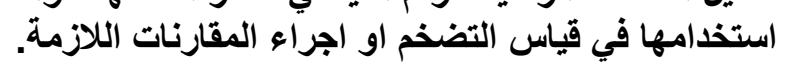

1-Box G, E.P \& Jenkins, G. M., 1976, "Time series analysis forcasting and control sanfrancisco Helden-day".

2-Box, G. E. and Tiaa, 1979, "Distribution of Residual Autocorrelation in Multiple Autoregressive", JASA, Vol. (74), pp. (928-934).

3-Hungarian Central Statistical Office, July 2007, "Seasonal Adjustment Methods \&Practices".

4- International Lab our Organization/International Monetary Fund/Organization for Economic Cooperation and Development/Statistical Office of the European Communities/United Nations/Reconstruction and Development/The World Bank ,2004," Consumer Price Index Manual".

5- International Lab our Organization/International Monetary Fund/Organization for Economic Cooperation and Development/Statistical Office of the European Communities/United Nations/Reconstruction and Development/The World Bank, 2004 ," Produce Price Index Manual".

6- Peter Brockwel ;Richard Davis(2002)."Introduction To Time Series \& Forecasting(2nd.ed)Spring er.p.36.1sBnNo-387-94719

7- Wei,W.S.(1990),"Time series Analysis",Addison-Wesly publishing company INC,USA

8- William R.Bell and Steven C. Hillmer,(1984) (Seasonal Adjustment of Economic Time Series) Statistical Research Series 


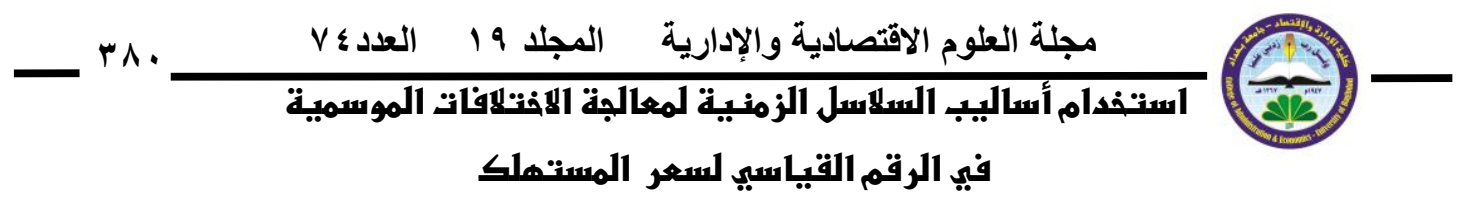

\title{
Using Time Series Methods To Modify The Seasonal Variations in the Consumer Price Index
}

\begin{abstract}
As is known that the consumer price index (CPI) is one of the most important price indices because of its direct effect on the welfare of the individual and his living.

We have been address the problem of Strongly seasonal commodities in calculating (CPI) and identifying some of the solution.

We have used an actual data for a set of commodities (including strongly seasonal commodities) to calculate the index price by using (Annual Basket With Carry Forward Prices method). Although this method can be successfully used in the context of seasonal commodities the index does not get rid of the tremendous season fluctuations .

In order to use (CPI) in measuring the general inflation and monthly or quarterly comparison, we must first decompose the seasonal component and eliminate its effect on the (CPI) series to get a seasonal adjusted series of (CPI) .

Many statistical methods are used to analysis (CPI) series, and one of these methods is the method of time series that takes into account the seasonal variations in the study of phenomena.

test to Ljung-Box We have used Box-Jenkens method in models building and then test the modesl, also we have found the seasonal adjusted series by using time series method
\end{abstract}

